\title{
THE MORPHOLOGIES AND ALIGNMENTS OF GAS, MASS, AND THE CENTRAL GALAXIES OF CLASH
} CLUSTERS OF GALAXIES

\author{
Megan Donahue $^{1}$, Stefano Ettori ${ }^{2}$, Elena Rasia ${ }^{3,4}$, Jack SaYers $^{5}$, Adi Zitrin ${ }^{5,9}$, Massimo Meneghetti ${ }^{2,5}$, G. Mark Voit ${ }^{1}$, \\ Sunil Golwala ${ }_{1}^{5}$, Nicole Czakon ${ }^{6}$, Gustavo Yepes ${ }^{7}$, Alessandro Baldi ${ }^{1}$, Anton Koekemoer ${ }^{8}$, and Marc Postman ${ }^{8}$ \\ Physics and Astronomy Dept., Michigan State University, East Lansing, MI, 48824 USA; donahue@ pa.msu.edu \\ ${ }^{2}$ Osservatori Astronomico di Bologna, Via Ranzani 1, 40127 Bologna, Italy; INFN, Sezione di Bologna, viale Berti Pichat 6/2, I-40127 Bologna, Italy \\ ${ }^{3}$ Department of Physics, University of Michigan, Ann Arbor, MI, 48109, USA \\ ${ }^{4}$ INAF-Osservatorio Astronomico of Trieste, via Tiepolo 11, I-34121 Trieste, Italy \\ ${ }^{5}$ Division of Physics, Math, and Astronomy, California Institute of Technology, Pasadena, CA 91125, USA \\ ${ }^{6}$ Institute of Astronomy and Astrophysics, Academia Sinica, No. 1, Section 4, Roosevelt Rd, Taipei 10617, Taiwan, ROC \\ ${ }^{7}$ Departamento de Física Teórica, Universidad Autónoma de Madrid, Cantoblanco, E-28049, Madrid, Spain \\ ${ }^{8}$ Space Telescope Science Institute, 3700 San Martin Drive, Baltimore, MD, 21218, USA \\ Received 2015 October 6; accepted 2016 January 11; published 2016 February 25
}

\begin{abstract}
Morphology is often used to infer the state of relaxation of galaxy clusters. The regularity, symmetry, and degree to which a cluster is centrally concentrated inform quantitative measures of cluster morphology. The Cluster Lensing and Supernova survey with Hubble Space Telescope (CLASH) used weak and strong lensing to measure the distribution of matter within a sample of 25 clusters, 20 of which were deemed to be "relaxed" based on their X-ray morphology and alignment of the X-ray emission with the Brightest Cluster Galaxy. Toward a quantitative characterization of this important sample of clusters, we present uniformly estimated X-ray morphological statistics for all 25 CLASH clusters. We compare X-ray morphologies of CLASH clusters with those identically measured for a large sample of simulated clusters from the MUSIC-2 simulations, selected by mass. We confirm a threshold in X-ray surface brightness concentration of $C \gtrsim 0.4$ for cool-core clusters, where $C$ is the ratio of X-ray emission inside $100 h_{70}^{-1} \mathrm{kpc}$ compared to inside $500 h_{70}^{-1} \mathrm{kpc}$. We report and compare morphologies of these clusters inferred from Sunyaev-Zeldovich Effect (SZE) maps of the hot gas and in from projected mass maps based on strong and weak lensing. We find a strong agreement in alignments of the orientation of major axes for the lensing, X-ray, and SZE maps of nearly all of the CLASH clusters at radii of $500 \mathrm{kpc}$ (approximately $1 / 2 R_{500}$ for these clusters). We also find a striking alignment of clusters shapes at the $500 \mathrm{kpc}$ scale, as measured with X-ray, SZE, and lensing, with that of the near-infrared stellar light at $10 \mathrm{kpc}$ scales for the 20 "relaxed" clusters. This strong alignment indicates a powerful coupling between the cluster- and galaxy-scale galaxy formation processes.
\end{abstract}

Key words: dark matter - galaxies: clusters: intracluster medium - galaxies: elliptical and lenticular, cD gravitational lensing: strong - gravitational lensing: weak - large-scale structure of universe

\section{BACKGROUND: CLUSTER MORPHOLOGY}

Clusters of galaxies represent the largest gravitationally bound systems in the universe, and their gravitational potentials are dominated by dark matter $(\sim 85 \%)$ (e.g., Voit 2005; Vikhlinin et al. 2006). The projected mass density of a cluster can be inferred from measurements of the distortion, statistical shear and magnification that gravitational lensing induces in background galaxies (e.g., Tyson et al. 1990). The gravitational potential also binds hot, X-ray emitting intracluster gas to the cluster (e.g., Forman \& Jones 1982). In a relatively relaxed cluster, the shape and depth of the gravitational potential and the entropy distribution of the gas completely determine its distribution in space and temperature (Voit \& Bryan 2001). X-ray and Sunyaev-Zeldovich (Sunyaev \& Zeldovich 1972) observations of relaxed clusters therefore trace the shape, centroid, and slope of the gravitational potential, but dynamical interactions can produce shocks or pressure waves that disturb the gas and complicate the relationship between the gas distribution and the gravitational potential (Wik et al. 2008). Historically, the locations and redshifts of the cluster galaxies themselves have been used to infer a projected model for the distribution of matter in the cluster, which then can be compared to a three-dimensional model inferred from the

\footnotetext{
${ }^{9}$ Hubble Fellow.
}

observations and analysis of the hot, X-ray emitting gas (Kent \& Sargent 1983; Fabricant et al. 1989). By combining multiple probes of the matter distribution in galaxy clusters we can minimize the dependence of our mass inferences on assumptions such as isotropy, symmetry, or hydrostatic equilibrium.

There is a rich history of classifying clusters of galaxies according to visual morphology. Abell (1958) did not provide morphological classifications of the clusters in his famous catalog, but Zwicky and his collaborators classified clusters in terms of their central concentration as compact, medium compact or open (e.g., Zwicky et al. 1961). Morgan (1961) divided a sample of 20 nearby Abell clusters into two classes, based on the types of galaxies in the cluster. This notion later developed into the Bautz-Morgan classification system (Bautz \& Morgan 1970), which distinguishes clusters by the presence of a dominant central galaxy (type I), the dominance of ellipticals but no single Brightest Cluster Galaxy (BCG) (type II), and the rest (type III). Correlations between the concentration, richness of a cluster, and its Bautz-Morgan type suggested a connection between the dynamic state of a cluster and its appearance (see review in Bahcall 1977).

Because of the potential connection to cosmological studies, there was great interest in the 1990s in trying to find robust methods of constraining the total matter density of the universe, the primordial power spectrum, or other cosmological 
parameters using morphological cues from clusters of galaxies (e.g., Binggeli 1982; de Theije et al. 1995; Splinter et al. 1997). These efforts ultimately proved unsatisfactory, in part because the galaxy counts used to define cluster shapes are prone to systematic uncertainties, even at relatively low redshifts. A more extended review of the morphological properties of clusters of galaxies can be found in Rasia et al. (2013b).

$\mathrm{X}$-ray imaging of the hot gas in clusters of galaxies provides a more straightforward means to reveal substructure and cluster shape. The intracluster gas represents some $\sim 85 \%$ of the baryons in the cluster. The X-ray emission from the intracluster gas, proportional to $n_{e}^{2}$ and a weaker function of temperature, is not as affected by projections and shot noise. Early X-ray images of clusters of galaxies from the Einstein satellite and their classification suggested a further connection between the prominence and centrality of the BCG and the dynamical state the system (Forman \& Jones 1982; Jones \& Forman 1984, 1999). X-ray imaging of the cluster gas in single Abell clusters revealed substructures undetected in the galaxy distribution on the sky but visible with dense redshift sampling (e.g., Beers et al. 1983). Identification of substructure in clusters may allow for increased purity of a sample of relaxed clusters. For example, Mantz et al. (2015) imposed rigorous selection based on cluster dynamical state in order to tightly constrain the cosmological evolution of the gas mass fraction of relaxed clusters. In this paper, we will discuss X-ray measurements made with the Advanced CCD Image Spectrometer (ACIS) on board the Chandra X-ray Observatory, which is capable of sub-arcsecond resolution of structures in X-ray emission from 10 million $\mathrm{K}$ gas.

The hot intergalactic gas in a cluster of galaxies can also be studied via its scattering signature on the cosmic microwave background (CMB) (Sunyaev \& Zeldovich 1972). This Sunyaev-Zeldovich effect (SZE) measures the frequencydependent shift in the $\mathrm{CMB}$ radiation intensity, induced by the interaction of the CMB photons with the hot intracluster electrons (Weymann 1965). The SZE scales with the electron pressure $\left(n_{e} T_{e}\right)$ integrated along the line of sight, and therefore provides a gas measurement that is nearly independent of the X-ray estimates, although often X-ray spectra are used to constrain gas temperatures. While the earliest measurements of this effect came from using beam-switching techniques with a single dish scanning across the cluster (e.g., Birkinshaw et al. 1978), SZE images were enabled by the use of interferometric arrays of radio telescopes, with most of the elements packed closely together to achieve short baselines to tease out the extended (arcminute-scale) signal (See review in Carlstrom et al. 2002). Currently, most SZE images are collected using large-format bolometric cameras, which are better than X-ray images at recovering emission from gas at large radii to and beyond the cluster virial radius (e.g., Plagge et al. 2010; Sayers et al. 2013) and are weighted more heavily toward larger radii due to the weaker dependence of the SZE signal on electron density.

The association of radio halos with clusters displaying irregular X-ray morphology provided support for the idea that a cluster's X-ray appearance can be used to discriminate between regular (relaxed) clusters and disturbed (dynamically active) ones (Rasia et al. 2013b and references therein). Cassano et al. (2010) used quantitative methods applied to the X-ray surface brightness distribution, such as the measure of the centroid shift, the concentration parameter and the third-order power ratio, to characterize substructures in a statistical sample of 32 $\mathrm{X}$-ray luminous galaxy clusters, with available radio (GMRT and/or VLA) observations. They showed that giant radio halos prefer to be associated with dynamically disturbed galaxy clusters, characterized by high values of the X-ray centroid shift and third power ratio moment, and low values of the concentration parameter. Cuciti et al. (2015), by studying a mass-selected sample of 75 galaxy clusters from the Planck SZE catalog in the redshift range $0.08<z<0.33$, confirmed that the presence of radio halos is associated with merging systems, defined according to X-ray morphology.

The purpose of the present work is: (i) to present and document morphological measurements of the CLASH clusters; (ii) to analyze the correlations among their morphological parameters in different spectral bands; (iii) to verify whether their morphologies are typical for CLASH-like massive systems in numerical simulations; (iv) to quantify the alignment between a cluster's X-ray appearance, its SZE appearance, and its projected mass density as inferred from gravitational lensing; and (v) to assess the alignment of BCGs at small radial scales with the larger-scale morphology of the cluster.

\section{THE CLASH PROJECT AND SAMPLE}

The CLASH cluster program and strategy are described in Postman et al. (2012a). Relevant cluster properties are provided in Table 1. CLASH was a Hubble Multi-Cycle Treasury program with multiple science goals. The most relevant CLASH science goal for this work was to obtain wellconstrained gravitational-lensing mass profiles for a sample of 25 massive clusters of galaxies between redshifts of 0.2-0.9. To avoid any biases that would be introduced by selecting clusters on the basis of their lensing signal, twenty of the CLASH clusters were instead selected on the basis of X-ray morphology, to have relatively round X-ray isophotes centered on a prominent BCG. The remaining five were selected to be systems capable of providing extraordinary, gravitationallyboosted views of the high redshift universe. All of the clusters have relatively hot intracluster gas (ICM), with global gas temperatures of $k T>5 \mathrm{keV}$. This program was allocated 524 orbits over a 3 year (cycle) period between May, 2010 and May, 2013. During this time, HST observed all 25 clusters with up to 16 passbands, utilizing the Wide Field Camera 3 (WFC3) Infrared (IR) and UV/Visible (UVIS) channels and the Advanced Camera for Surveys (ACS). All CLASH clusters also have good to excellent Chandra X-ray data, with at least $6000-10,000 \mathrm{X}$-ray events between 0.5 and $7.0 \mathrm{keV}$ in publicly available datasets.

Observations of the CLASH cluster sample have already shown that their concentration-mass relation is consistent with $\Lambda$-CDM based predictions, once the CLASH X-ray morphological selection is taken into account (Meneghetti et al. 2014; Umetsu et al. 2014, 2015; Merten et al. 2015). This major result confirmed suspicions that previous studies, having selected clusters with prominent lensing features, gave mass concentration measurements biased higher than those predicted from simulations based on idealized mass-selection of clusters. This effect was suggested in (e.g., Comerford \& Natarajan 2007; Meneghetti et al. 2010; Groener et al. 2016). For the CLASH analysis, biases induced by X-ray morphological selection were quantified by selecting clusters from simulations in the same way that they were selected for inclusion in the CLASH 
Table 1

CLASH Clusters and Chandra X-Ray Observations

\begin{tabular}{|c|c|c|c|c|c|}
\hline $\begin{array}{l}\text { Cluster Name } \\
(-)\end{array}$ & $\begin{array}{c}\text { R.A. } \\
\text { (hh:mm:ss) }\end{array}$ & $\begin{array}{c}\text { Decl. } \\
\text { (dd:mm:ss) }\end{array}$ & $(-)$ & $\begin{array}{l}0.5 R_{500} \\
(\operatorname{arcsec})\end{array}$ & $\begin{array}{c}\text { Chandra } \\
\text { Obs ID }\end{array}$ \\
\hline Abell 209 & $01: 31: 52.54$ & $-13: 36: 40.4$ & 0.206 & 203 & 3579,522 \\
\hline Abell 383 & $02: 48: 03.40$ & $-03: 31: 44.9$ & 0.187 & 219 & 2321 \\
\hline MACSJ0329-02 & $03: 29: 41.56$ & $-02: 11: 46.1$ & 0.450 & 101 & 6108,3582 \\
\hline MACSJ0416-24 & 04:16:08.38 & $-24: 04: 20.8$ & 0.396 & $\ldots$ & 10446 \\
\hline MACSJ0429-02 & $04: 29: 36.05$ & $-02: 53: 06.1$ & 0.399 & 113 & 3271 \\
\hline MACSJ0647+70 & $06: 47: 50.27$ & $+70: 14: 55.0$ & 0.584 & $\ldots$ & 3584,3196 \\
\hline MACSJ0717+37 & $07: 17: 32.63$ & $+37: 44: 59.7$ & 0.548 & $\ldots$ & 4200 \\
\hline MACSJ0744+39 & $07: 44: 52.82$ & $+39: 27: 26.9$ & 0.686 & 74 & 6111 \\
\hline Abell 611 & 08:00:56.82 & $+36: 03: 23.6$ & 0.288 & 149 & 3194 \\
\hline MACSJ1115+01 & $11: 15: 51.90$ & $+01: 29: 55.1$ & 0.355 & 124 & 9375 \\
\hline MACSJ1149+22 & $11: 49: 35.69$ & $+22: 23: 54.6$ & 0.544 & $\ldots$ & 3589,1656 \\
\hline Abell 1423 & $11: 57: 17.36$ & $+33: 36: 37.47$ & 0.213 & $\ldots$ & 11724 \\
\hline MACSJ1206-08 & $12: 06: 12.09$ & $-08: 48: 04.4$ & 0.439 & 109 & 3277 \\
\hline CLJ1226+3332 & $12: 26: 58.25$ & $+33: 32: 48.6$ & 0.890 & 81 & $5014,3180,932$ \\
\hline MACSJ1311-03 & 13:11:01.80 & $-03: 10: 39.8$ & 0.494 & 81 & 6110,9381 \\
\hline RXJ1347-1145 & $13: 47: 30.62$ & $-11: 45: 09.4$ & 0.451 & 117 & 3592 \\
\hline MACSJ1423+24 & $14: 23: 47.88$ & $+24: 04: 42.5$ & 0.545 & 82 & 4195 \\
\hline MACSJ1532+30 & $15: 32: 53.78$ & $+30: 20: 59.4$ & 0.363 & 105 & 1665,1649 \\
\hline MACSJ1720+35 & $17: 20: 16.78$ & $+35: 36: 26.5$ & 0.387 & 115 & 6107 \\
\hline Abell 2261 & $17: 22: 27.18$ & $+32: 07: 57.3$ & 0.224 & 217 & 5007 \\
\hline MACSJ1931-26 & $19: 31: 49.62$ & $-26: 34: 32.9$ & 0.352 & 117 & 3282,9382 \\
\hline MACSJ2129-07 & $21: 29: 26.06$ & $-07: 41: 28.8$ & 0.570 & $\ldots$ & 3595,3199 \\
\hline RXJ2129+0005 & $21: 29: 39.96$ & $+00: 05: 21.2$ & 0.234 & 161 & 552,9370 \\
\hline MS2137-2353 & $21: 40: 15.17$ & $-23: 39: 40.2$ & 0.313 & 148 & 4974,5250 \\
\hline RXJ2248-4431 & $22: 48: 43.96$ & $-44: 31: 51.3$ & 0.348 & 141 & 4966 \\
\hline
\end{tabular}

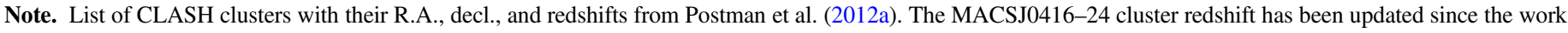

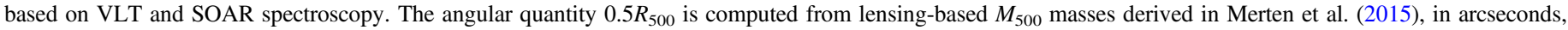
assuming the cosmology assumed in that work.

sample. Quantified X-ray morphologies were measured from maps for the simulated clusters with procedures identical to those used on the actual X-ray data (Meneghetti et al. 2014).

In this paper, we provide quantitative X-ray surface-brightness morphological parameters for the 25 clusters in the CLASH survey (Postman et al. 2012b), as well as similarlydefined morphological parameters derived from gravitational lensing (shear) projected mass maps and SZE gas (Compton " $y$ "-parameter) maps. Two sets of morphological measurements were made, one inside a fixed metric aperture of 500 $h_{70}^{-1} \mathrm{kpc}$ and the other inside half the $R_{500}$ overdensity radius (i.e., $R_{500}$ is the radius inside which the average mass density is 500 times the critical density at the redshift of the cluster, so it is a cosmology- and mass-dependent quantity.) We reconstructed the angular scale corresponding to $0.5 R_{500}$ in arcseconds from the $M_{500} h^{-1}$ mass quantity reported in Merten et al. (2015). To avoid ambiguity, we report that specific angular scale for each CLASH cluster in Table 1. The fixed metric aperture of $500 \mathrm{kpc}$ has the advantage of not changing significantly from analysis to analysis, as well as having been used by previous observers for similar purposes (e.g., Jeltema et al. 2005; Cassano et al. 2010). For the CLASH sample, a fixed metric aperture is approximately the same fraction of the virial radius for most of the clusters, since these clusters are similar in mass. Regardless of the aperture we use, the morphologies of the BCGs at kpc scales in the relaxed sample are strikingly aligned with gravitational potential elongations within these much larger apertures, suggesting a strong relationship between the assembly of the BCG and the cluster as a whole.
We also compare CLASH clusters to a broader sample of simulated clusters from MUSIC-2 ${ }^{10}$ (Sembolini et al. 2013), selected to have similar masses by making a cut at a minimum global temperature of $5 \mathrm{keV}$, without regard to morphology. The purpose of this comparison is to examine how typical the morphological properties of CLASH clusters are of massive, simulated clusters in any dynamical state. Throughout the paper, we assume cosmological parameters of $\Omega_{M}=0.3$, $\Omega_{\Lambda}=0.7$, and $H_{0}=70 h_{70} \mathrm{~km} \mathrm{~s}^{-1} \mathrm{Mpc}^{-1}$ (i.e., $h_{70}=1$ is the default).

\section{DATA PROCESSING}

\subsection{X-Ray Imaging}

We use X-ray events from the Chandra X-ray Observatory, processed and filtered as described in Donahue et al. (2014) (CALDB v4.5.9, CIAO v4.6). The data sets are tabulated in Table 1. Binned X-ray maps were generated with $2 \times 2$ instrument pixel (0.!984) spatial bins with the CIAO script fluximage. Two exposure-corrected images were constructed for each observation ID, $0.7-2.0 \mathrm{keV}$ soft band and a $0.7-8.0 \mathrm{keV}$ broad band. The exposure maps were based on the best bad-pixel maps, aspect solutions and mask files available, and assumed average energies of 1.0 and $2.0 \mathrm{keV}$, respectively. Adaptively smoothed images of the central $300 \times 300 \mathrm{kpc}$ from the exposure-corrected $0.7-8.0 \mathrm{keV}$ broad band images are shown in Figure 1. The the morphological measurements were made on images in $0.7-2.0 \mathrm{keV}$ band, for

\footnotetext{
${ }^{10}$ MUSIC website: http://music.ft.uam.es
} 

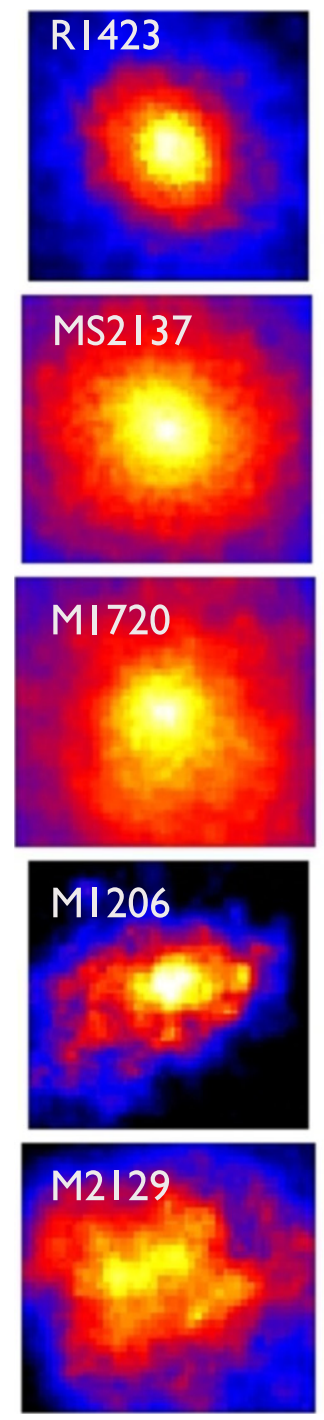
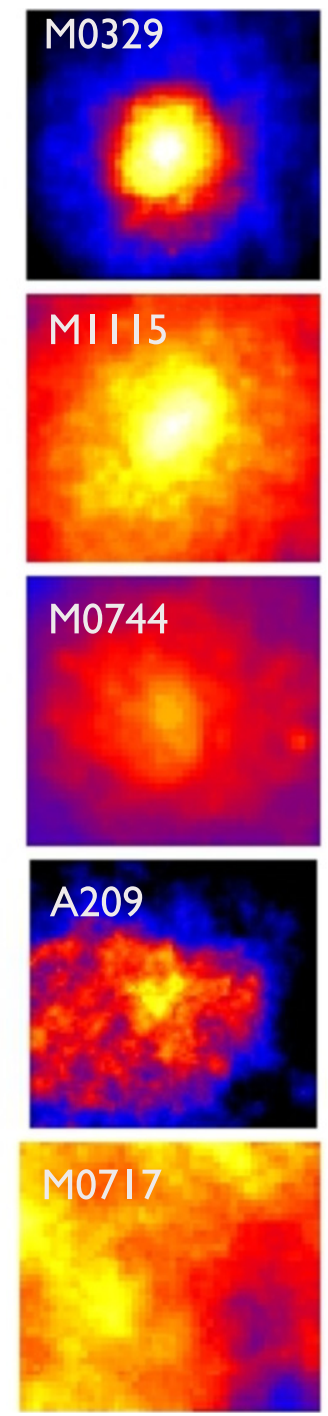
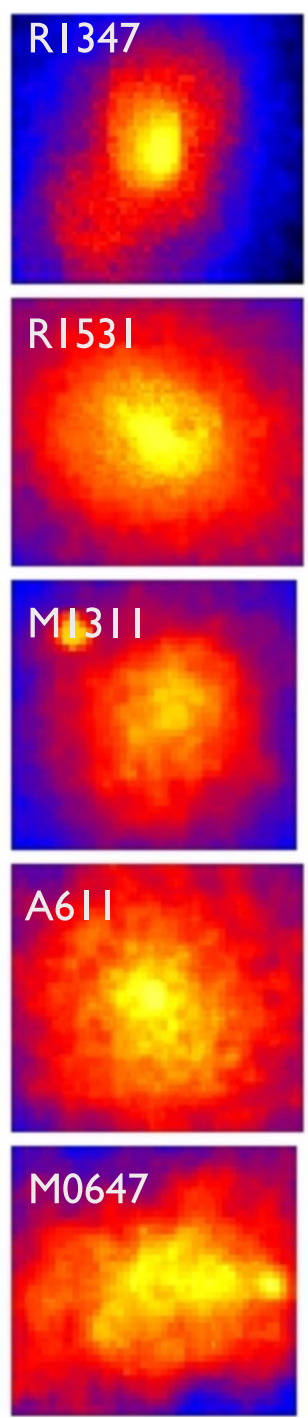
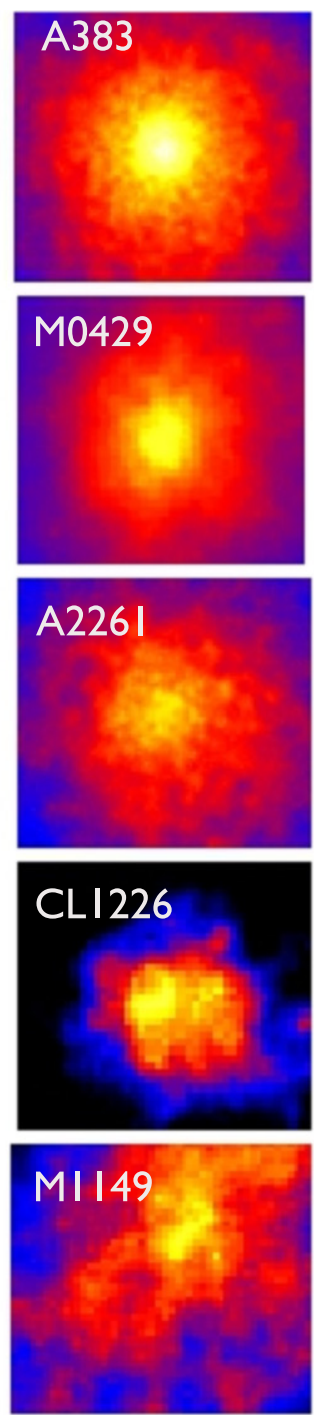
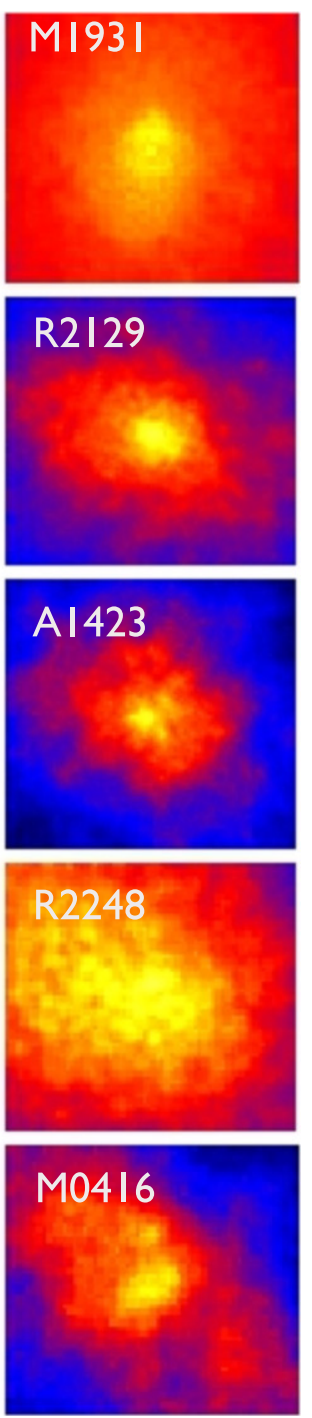

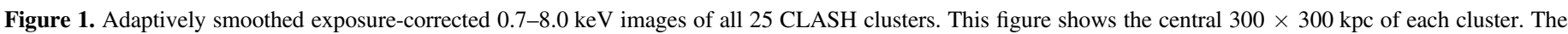

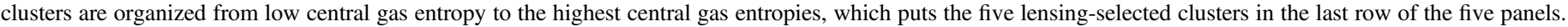

the most optimal signal-to-noise and to minimize the X-ray emission sensitivity to temperature variations.

\subsection{SZE Imaging}

The Bolocam SZE images were downloaded from NASA's Infrared Science Archive (IRSA). ${ }^{11}$ The details of these data are given in Sayers et al. (2013) and Czakon et al. (2015). Specifically, we made use of the data in the file unfiltered_image.fits, which provides an image of the cluster that are corrected from the distortion of atmospheric noise filtering, and therefore are well suited to constraining morphological parameters. To characterize the noise in these images, which is correlated between pixels, we made use of the 1000 bootstrap noise realizations contained in the file unfiltered_image_noise_realizations.fits. The images are $10^{\prime} \times 10^{\prime}$ in size, and the Bolocam point-spread function (PSF) has a full width half maximum (FWHM) of 58". The SZE brightness and noise varies over the CLASH sample, and the peak signal-to-noise

11 irsa.ipac.caltech.edu/data/Planck/release_2/ancillary-data/bolocam/ ratio $(\mathrm{S} / \mathrm{N})$ per resolution element in the images ranges from 5 to 40 .

\subsection{Lensing Models and Maps}

Gravitational lensing maps of the surface mass density $(\kappa)$ have been constructed utilizing both strong- and weak-lensing information from the Hubble Space Telescope. The analysis is described more fully in Zitrin et al. (2015), and here we give a very brief summary. The lens modeling was performed using two complementary parametric methods, to obtain a better grasp on systematics. The light-traces-mass (LTM) method assumes that light traces mass for both the galaxies and the dark matter, with the latter being a smooth version of the former, and the two components are added with a free relative weight. The second method (PIEDeNFW) assumes LTM for the cluster galaxies but then fits an analytical elliptical NFW form (Navarro et al. 1997) for the dark matter (PIEMDeNFW: Pseudo-Isothermal Elliptical Mass Distribution plus elliptical Navarro Frenk and White profile). The minimization is performed via a Monte Carlo Markov Chain. The lensing maps of the best fit model are then generated on a grid similar 
to the original HST image used to define the input, with a spatial resolution of 65 milliarsceconds per pixel. Statistical uncertainties were estimated from 100 random steps from the MC chain. We use the second method (PIEMDeNFW) for the lensing map for our baseline analysis, and we estimate systematic uncertainties by comparing our baseline result to an identical analysis using the LTM method.

Two interesting findings from Zitrin et al. (2015) are worth noting here. The first is that systematic uncertainties dominate the lensing error budget and are about $40 \%$ in $\kappa$, on average, per pixel, among all CLASH clusters. Zitrin et al. (2015) determine that typical errors on lensing quantities are thus underestimated, due to traditional use of only one method per cluster. Efforts have been made in the past couple of years to overcome this and learn about systematics in lens- and stronglens modeling in particular. However, various factors of uncertainty such as errors from large scale structure or other correlated matter along the line of sight (e.g., D'Aloisio \& Natarajan 2011; Host 2012) are sometimes not accounted for properly in error budgets. Zitrin et al. (2015) therefore recommend, in using these maps, to estimate the typical systematic error from the differences between the two methods they employ. These systematic differences in $\kappa$ do not lead to significant variance in our scientific conclusions.

\subsection{Simulations and Mock Cluster Catalogs}

The MUSIC-2 sample includes 282 clusters selected within a cosmological dark-matter box of volume $\left(1 h^{-1} \mathrm{Gpc}\right)^{3}$ and resimulated with a hydro component at higher resolution (Sembolini et al. 2013). The parent simulation (the MultiDark simulation) was carried out with the code ART (Kravtsov et al. 1997) and contained $2048^{3}$ particles. The underlying cosmological model is identical to what we have assumed in this paper. They assume a flat universe described by the following values of the cosmological parameters: $\Omega_{M}=0.3$ as matter density; $\Omega_{b}=0.0469$ as baryon density; $\sigma_{8}=0.82$ as primordial amplitude of fluctuation in a scale of $8 \mathrm{~h}^{-1} \mathrm{Mpc}$; $n=0.95$ as power spectrum index; and $h=0.7$ as reduced Hubble parameter $\left(h_{70}=1\right)$. The CLASH sample is comprised of clusters with gas temperatures above $5 \mathrm{keV}$. Assuming $M-T$ relations from Vikhlinin et al. (2009), this temperature limit corresponds to the a mass limit equal to $M_{500}>3.5 \times 10^{14} M_{\odot} h^{-1}$, which is satisfied by $\sim 100$ clusters at $z=0.333$ (only 1 simulated cluster has mass $M_{500}>10^{15} M_{\odot} h^{-1}$ at that redshift).

The re-simulations involved Lagrangian regions of $6 \mathrm{~h}^{-1}$ $\mathrm{Mpc}$ radius around the most massive halos (with virial masses above $10^{15} h^{-1} M_{\odot}$ at redshift $z=0$ ) and were performed by using the TREEPM+SPH code GADGET (Springel 2005). Two sets of re-simulations were produced including both nonradiative and radiative physics. In this work, as in Meneghetti et al. (2014), we focus on the non-radiative simulation because the radiative run did not include any prescription for feedback by active galactic nuclei, implying that the cluster core is affected by over-cooling (Borgani \& Kravtsov 2011). In the radiative case, the condensation of X-ray luminous gas in the center is extreme (Rasia et al. 2013a), the light concentration is not realistic, and the cluster isophotal shapes are less in agreement with observations than non-radiative simulations (Lau et al. 2012). We note that in a comparison project of various codes and AGN-feedback implementation schemes (Sembolini et al. 2015), that most of the simulations that include AGN feedback give similar dark matter distributions and gas fractions as those found in the non-radiative simulations outside of cluster cores.

The mass resolution of the MUSIC-2 simulations corresponds to $m_{\mathrm{DM}}=9.01 \times 10^{8} h^{-1} M_{\odot}$ for the dark-matter particles and $m_{\text {gas }}=1.9 \times 10^{8} h^{-1} M_{\odot}$ for the gas particles. The gravitational softening was set to $6 h^{-1} \mathrm{kpc}$. Ten different simulation snapshots were stored. We analyze those four that cover the same redshift range of the CLASH sample: $z=0.25$, $0.33,0.43,0.67$, respectively.

\subsubsection{Mock X-Ray Catalog}

For each simulated halo, we produce three Chandra-like event files corresponding to the orthogonal line-of-sight projectionsof the original cosmological volume. These projections are therefore randomly oriented relative to a cluster. The three files are not co-added and are analyzed independently. The tool adopted is the X-ray MAp Simulator (X-MAS, Gardini et al. 2004; Rasia et al. 2008) which accounts for the ancillary response function and the redistribution matrix function of the ACIS-S3 detector on board of the Chandra satellite. The field of view is set equal to 16 arcmin, which corresponds to $\sim 4.5 \mathrm{Mpc}$ at $z=0.333$. The two outer radii used in the current analysis, $500 \mathrm{kpc}$ and $0.5 R_{500}$, are amply within the mock X-ray image. To generate the event files we assume a fixed metallicity with value equal to 0.3 solar (as tabulated by Anders \& Grevesse 1989); we include the galactic absorption with a WABS model $\left(N_{H}=5 \times 10^{20} \mathrm{~cm}^{-2}\right)$; and we impose an exposure time equal to $100 \mathrm{ks}$.

As we have done for the real X-ray observations, to evaluate the morphological parameters from the mock catalog, we produce soft X-ray band ([0.5-2] keV) images binned by $2 \times 2$ $\operatorname{arcsec}^{2}$. For a detailed description of the method, see Rasia et al. (2013c).

\subsubsection{Mock SZE Catalog}

To evaluate the effect of the Bolocam PSF on the morphological parameters (see Section 4.2), we produce maps of the Compton $y$ parameter. For each simulated cluster, we chose only one line of sight, centered the map on the minimum of the potential well, and similarly to the X-ray images consider a field of view of 16 arcmin as a side and an integrating distance of $10 \mathrm{Mpc}$. The resolution of each pixel is 1 arcsec.

\section{MORPHOLOGY MEASUREMENTS}

\subsection{Morphologies}

For the following discussion we define general quantities based on analysis of maps of scalar observables.In the discussion to follow, we will talk in terms of the surface brightness of light. However, we also map the SZE Compton y-parameter or surface mass density for a cluster and characterize the distribution using identical conventions applied to maps of the light distribution. In this study, we quantify 2D cluster morphology using the following parameters:

1. Concentration, $C$ is defined here to be the ratio between the light (or other mapped observable) within a circular aperture with a radius $R_{\text {inner }}$ and the total light enclosed within a circular aperture with a radius $R_{\text {outer }}$ The concentration $C$ is defined where $\left(R_{\text {inner }}, R_{\text {outer }}\right)=$ $(100,500) \mathrm{kpc}$ (See also Cassano et al. 2010). For the 
case where we use scaled apertures to define the radii, $\left(R_{\text {inner }}, R_{\text {outer }}\right)=(0.15,0.5) R_{500}$. Note that this $C$ is $n o t$ the same concentration $c$ used in the mass-concentration relation, nor is it based on the percentage of total enclosed light, as is occasionally used elsewhere for galaxies and X-ray clusters (e.g., Abraham et al. 1994).

2. Centroid shift, w (e.g., O'Hara et al. 2006; Poole et al. 2006; Maughan et al. 2008; Ventimiglia et al. 2008; Böhringer et al. 2010; Cassano et al. 2010; Rasia et al. 2013c) is the standard deviation of the projected separation between the X-ray peak and centroids estimated within circular apertures of increasing radius up to $R_{\max }=500 h_{70}^{-1} \mathrm{kpc}$ :

$$
w=\left[\frac{1}{N-1} \sum_{i}\left(\Delta_{i}-\bar{\Delta}\right)^{2}\right]^{1 / 2} \frac{1}{R_{\max }}
$$

3. Power ratio, $P_{3} / P_{0} \equiv P 30, P_{4} / P_{0} \equiv P 40$ : the power ratios are defined from the multipole decomposition of the two-dimensional X-ray surface brightness in circular apertures centered on the cluster's centroid:

$$
\frac{P_{m}}{P_{0}}=\frac{\left(a_{m}^{2}+b_{m}^{2}\right) /\left(2 m^{2} R^{2 m}\right)}{\left[a_{0} \ln (R)\right]^{2}}
$$

where $\quad a_{m}(R)=\int_{R<R_{\max }} S(\boldsymbol{x}) R^{m} \cos (m \phi) d^{2} x$, $b_{m}(R)=\int_{R<R_{\max }} S(\boldsymbol{x}) R^{m} \sin (m \phi) d^{2} x, \boldsymbol{x}=(R, \phi), S(\boldsymbol{x})$ is the X-ray surface brightness at sky location $x$. The ratio $P_{3} / P_{0}$, estimated from the above equations with $m=3$, is sensitive to deviations from mirror symmetry and insensitive to ellipticity, in the sense that a high value of $P_{3} / P_{0}$ indicates a clear asymmetric structure in the ICM (See e.g., Buote \& Tsai 1995, 1996; Jeltema et al. 2005; Ventimiglia et al. 2008; Böhringer et al. 2010; Cassano et al. 2010; Rasia et al. 2013c.) Power ratios for clusters of galaxies are typically quite tiny $\left(\sim 10^{-7}\right)$ with statistical uncertainties not much smaller, but they span a significant dynamic range (4 orders of magnitude in the case of CLASH clusters).

4. Axial ratio (AR) is a measure of the elongation of the cluster surface-brightness map. We use the same procedure as documented in (Donahue et al. 2015). Briefly, the AR is estimated from the ratio of the non-zero elements after the diagonalization of a symmetric $2 \times 2$ matrix with elements equal to the second moments of the surface brightness, computed at each pixel element $(x, y)$ within the given aperture centered on the centroid. AR is defined as the ratio between the lengths of the major and minor axes, with values between 0.0 and $1.0(\mathrm{AR}=1.0$ corresponds to the circular case).

5. Position angle, (PA), by astronomical convention, is the alignment of the semimajor axis in degrees East of North. It is computed simultaneously with the AR (Donahue et al. 2015). Briefly, it is the rotation angle in degrees required to bring the matrix of the second moments into its diagonal form, and we correct this formal angle to the astronomical convention. ${ }^{12}$ Because of the degeneracy of rotation of the long axis, we occasionally add or subtract $180^{\circ}$ in plots in order to more easily compare PA

\footnotetext{
12 The IDL routine ELLFIT, which uses the same conventions described here, was used to derive eccentricity/ axial ratio and the position angle. http://www. astro.washington.edu/docs/idl/cgi-bin/getpro/library09.html?ELLFIT.
}

Table 2

\begin{tabular}{|c|c|c|c|}
\hline Quantity & Sample & Mean & $N$ \\
\hline$C 500 \mathrm{kpc}$ & X-ray & $0.43 \pm 0.13$ & 20 \\
\hline$C \mathrm{r} 500 / 2$ & X-ray* & $0.48 \pm 0.12$ & 19 \\
\hline$C 500 \mathrm{kpc}$ & all & $0.37 \pm 0.16$ & 25 \\
\hline C500 kpc & SL & $0.11 \pm 0.08$ & 5 \\
\hline$w 500 \mathrm{kpc}$ & $\mathrm{X}$-ray & $0.005 \pm 0.010$ & 20 \\
\hline$w \mathrm{r} 500 / 2$ & X-ray* & $0.005 \pm 0.008$ & 19 \\
\hline$w 500 \mathrm{kpc}$ & all & $0.006 \pm 0.012$ & 25 \\
\hline$w 500 \mathrm{kpc}$ & SL & $0.020 \pm 0.010$ & 5 \\
\hline Log P30 $500 \mathrm{kpc}$ & $\mathrm{X}$-ray & $-7.03 \pm 0.46$ & 20 \\
\hline Log P30 r500/2 & X-ray* & $-7.30 \pm 0.60$ & 19 \\
\hline Log P30 $500 \mathrm{kpc}$ & all & $-6.90 \pm 0.66$ & 25 \\
\hline Log P30 $500 \mathrm{kpc}$ & SL & $-5.80 \pm 0.40$ & 5 \\
\hline Log P40 $500 \mathrm{kpc}$ & $\mathrm{X}$-ray & $-7.45 \pm 0.46$ & 20 \\
\hline Log P40 r500/2 & X-ray* & $-7.62 \pm 0.52$ & 19 \\
\hline Log P40 $500 \mathrm{kpc}$ & all & $-7.35 \pm 0.63$ & 25 \\
\hline Log P40 $500 \mathrm{kpc}$ & SL & $-6.25 \pm 0.56$ & 5 \\
\hline Axial Ratio $500 \mathrm{kpc}$ & $\mathrm{X}$-ray & $0.91 \pm 0.05$ & 20 \\
\hline Axial Ratio r500/2 & X-ray* & $0.89 \pm 0.05$ & 19 \\
\hline Axial Ratio $500 \mathrm{kpc}$ & all & $0.90 \pm 0.06$ & 25 \\
\hline Axial Ratio 500 kpc & SL & $0.81 \pm 0.06$ & 5 \\
\hline PA Difference (Deg) & X-ray* & $0.9 \pm 11$ & 19 \\
\hline
\end{tabular}

Median X-Ray Morphologies for the CLASH Samples

Note. Medians and standard deviations of the X-ray based morphological quantities for subsamples of the CLASH clusters. The "all" sample is all 25 CLASH clusters. The "X-ray" sample is all 20 X-ray selected clusters measured at $500 \mathrm{kpc}$. The "X-ray", sample is the $19 \mathrm{X}$-ray selected clusters with measurements out to $0.5 R_{500}$. The "SL" sample is the 5 lensing-selected CLASH clusters. The PA difference reported in this table is the difference in degrees between the orientation of the longest axis, measured at $500 \mathrm{kpc}$ versus $0.5 R_{500}$, showing that the exact definition of the aperture does not affect the PA estimate.

measurements made for the same cluster, but from different maps.

\subsection{X-Ray Morphologies}

We estimated uncertainties in the morphological parameters by measuring multiple versions of the maps. The error budget for each X-ray morphological parameter was estimated by Monte-Carlo methods, by re-sampling the counts per pixel according to their Poissonian error to make statistically similar maps as in (Cassano et al. 2010).

The X-ray results are summarized and reported in Table 2. $\mathrm{X}$-ray results for individual clusters are reported in Table 3.

For all comparisons we make between the X-ray morphological parameters and the same parameters derived from the SZE, lensing, and simulated maps, we have chosen a fixed metric radius of $500 \mathrm{kpc}$. Ideally, we would choose a fixed fraction of a radius derived by a mass overdensity, such as half of the $R_{500}$ radius, which is defined to be the radius inside which the mean density is 500 times the critical density at the same redshift. However in practice, this radius can be difficult to work with in an analysis, since it is dependent on the mass estimate, and there are multiple technique-dependent mass measurements for any given cluster. For the CLASH clusters, $0.5 R_{500}$ is approximately $500 \mathrm{kpc}$ for each cluster. A $500 \mathrm{kpc}$ radius turns out to define an area sampled by all of the X-ray and SZE measurements without significant extrapolation. So for comparison of measurements of all $25 \mathrm{CLASH}$ clusters 
Table 3

X-Ray Morphological Properties for the CLASH Sample $500 \mathrm{kpc}$

\begin{tabular}{|c|c|c|c|c|c|c|c|c|c|c|c|c|c|c|}
\hline Name & $\begin{array}{c}\text { R.A. } \\
\text { Centroid }\end{array}$ & $\begin{array}{c}\text { Decl. } \\
\text { Centroid }\end{array}$ & $C$ & $\begin{array}{c}C \\
\text { unc }\end{array}$ & $w$ & $\begin{array}{c}w \\
\text { unc }\end{array}$ & P30 & $\begin{array}{l}\text { P30 } \\
\text { unc }\end{array}$ & P40 & $\begin{array}{l}\mathrm{P} 40 \\
\text { unc }\end{array}$ & $\mathrm{AR}$ & $\begin{array}{l}\text { AR } \\
\text { unc }\end{array}$ & PA & $\begin{array}{l}\text { PA } \\
\text { unc }\end{array}$ \\
\hline A209 & 22.97016 & -13.6118 & 0.167 & 0.005 & $5.2 \mathrm{E}-03$ & $1.0 \mathrm{E}-03$ & $4.8 \mathrm{E}-08$ & $5.6 \mathrm{E}-08$ & 3.78E-08 & 3.3E-08 & 0.91 & 0.01 & -49 & 3 \\
\hline A383 & 42.01374 & -3.52945 & 0.525 & 0.004 & $1.8 \mathrm{E}-03$ & 4.7E-04 & $5.8 \mathrm{E}-08$ & 2.7E-08 & $6.03 \mathrm{E}-09$ & $6.1 \mathrm{E}-09$ & 0.97 & 0.01 & 22 & 11 \\
\hline 0329-02 & 52.42264 & -2.19577 & 0.488 & 0.006 & $1.1 \mathrm{E}-02$ & $1.1 \mathrm{E}-03$ & $1.6 \mathrm{E}-07$ & $8.5 \mathrm{E}-08$ & $6.76 \mathrm{E}-08$ & $3.8 \mathrm{E}-08$ & 0.95 & 0.02 & -31 & 6 \\
\hline 0416-24 & 64.03614 & -24.07162 & 0.091 & 0.004 & $1.5 \mathrm{E}-02$ & $4.0 \mathrm{E}-03$ & $1.5 \mathrm{E}-06$ & 7.4E-07 & 8.54E-07 & $3.5 \mathrm{E}-07$ & 0.75 & 0.02 & 37 & 2 \\
\hline 0429-02 & 67.39994 & -2.88486 & 0.531 & 0.006 & $4.8 \mathrm{E}-03$ & $1.4 \mathrm{E}-03$ & $2.0 \mathrm{E}-07$ & $1.0 \mathrm{E}-07$ & 2.67E-08 & 2.0E-08 & 0.89 & 0.02 & -14 & 4 \\
\hline $0647+70$ & 101.95787 & 70.24891 & 0.242 & 0.008 & $1.0 \mathrm{E}-02$ & $2.3 \mathrm{E}-03$ & $6.4 \mathrm{E}-07$ & $3.1 \mathrm{E}-07$ & $5.68 \mathrm{E}-07$ & $2.8 \mathrm{E}-07$ & 0.76 & 0.02 & -80 & 2 \\
\hline $0717+37$ & 109.38513 & 37.75319 & 0.055 & 0.004 & $4.5 \mathrm{E}-02$ & $3.4 \mathrm{E}-03$ & $2.5 \mathrm{E}-06$ & 9.7E-07 & $9.75 \mathrm{E}-07$ & $4.1 \mathrm{E}-07$ & 0.84 & 0.02 & -29 & 2 \\
\hline $0744+39$ & 116.21812 & 39.45748 & 0.365 & 0.006 & 2.4E-02 & $1.6 \mathrm{E}-03$ & $1.4 \mathrm{E}-07$ & $1.2 \mathrm{E}-07$ & $2.81 \mathrm{E}-07$ & $1.0 \mathrm{E}-07$ & 0.98 & 0.01 & -22 & 37 \\
\hline A611 & 120.23689 & 36.05725 & 0.335 & 0.004 & $2.0 \mathrm{E}-03$ & 4.6E-04 & $8.0 \mathrm{E}-08$ & $4.0 \mathrm{E}-08$ & 2.33E-08 & $1.6 \mathrm{E}-08$ & 0.95 & 0.01 & 33 & 4 \\
\hline $1115+01$ & 168.96666 & 1.49813 & 0.430 & 0.008 & $5.6 \mathrm{E}-03$ & $1.7 \mathrm{E}-03$ & $1.9 \mathrm{E}-07$ & $1.2 \mathrm{E}-07$ & $1.68 \mathrm{E}-08$ & $2.5 \mathrm{E}-08$ & 0.85 & 0.03 & -29 & 3 \\
\hline $1149+22$ & 177.39769 & 22.4004 & 0.111 & 0.007 & $1.9 \mathrm{E}-02$ & $3.2 \mathrm{E}-03$ & $5.5 \mathrm{E}-06$ & $1.6 \mathrm{E}-06$ & $5.27 \mathrm{E}-07$ & 3.3E-07 & 0.81 & 0.02 & -36 & 2 \\
\hline A1423 & 179.32236 & 33.61042 & 0.287 & 0.004 & 4.6E-03 & $7.0 \mathrm{E}-04$ & $6.8 \mathrm{E}-08$ & $5.4 \mathrm{E}-08$ & 4.13E-08 & $3.0 \mathrm{E}-08$ & 0.91 & 0.02 & 55 & 3 \\
\hline $1206-08$ & 181.55339 & -8.8027 & 0.223 & 0.007 & $3.6 \mathrm{E}-02$ & $2.0 \mathrm{E}-03$ & 2.3E-06 & 4.4E-07 & 5.29E-07 & $1.4 \mathrm{E}-07$ & 0.85 & 0.01 & 10 & 2 \\
\hline $1226+33$ & 186.74102 & 33.54674 & 0.245 & 0.010 & $5.5 \mathrm{E}-03$ & $2.1 \mathrm{E}-03$ & $1.9 \mathrm{E}-07$ & $2.5 \mathrm{E}-07$ & $6.05 \mathrm{E}-08$ & 7.4E-08 & 0.95 & 0.03 & -69 & 13 \\
\hline $1311-03$ & 197.75704 & -3.17733 & 0.488 & 0.013 & $3.2 \mathrm{E}-03$ & $1.2 \mathrm{E}-03$ & $1.0 \mathrm{E}-07$ & $1.1 \mathrm{E}-07$ & $1.10 \mathrm{E}-07$ & 7.4E-08 & 0.89 & 0.04 & -16 & 6 \\
\hline $1347-1145$ & 206.87852 & -11.753 & 0.506 & 0.003 & $1.4 \mathrm{E}-02$ & $3.6 \mathrm{E}-04$ & $1.3 \mathrm{E}-07$ & $2.5 \mathrm{E}-08$ & $1.48 \mathrm{E}-08$ & 7.2E-09 & 0.84 & 0.01 & -1 & 1 \\
\hline $1423+24$ & 215.94964 & 24.07839 & 0.555 & 0.009 & $3.5 \mathrm{E}-03$ & $1.4 \mathrm{E}-03$ & $1.6 \mathrm{E}-07$ & $1.2 \mathrm{E}-07$ & $6.20 \mathrm{E}-08$ & $4.9 \mathrm{E}-08$ & 0.88 & 0.02 & 17 & 7 \\
\hline $1532+30$ & 233.22438 & 30.34978 & 0.571 & 0.007 & $1.9 \mathrm{E}-03$ & $8.3 \mathrm{E}-04$ & $1.2 \mathrm{E}-08$ & $1.3 \mathrm{E}-08$ & 2.64E-08 & $1.8 \mathrm{E}-08$ & 0.92 & 0.02 & 55 & 6 \\
\hline $1720+35$ & 260.06941 & 35.60649 & 0.417 & 0.008 & $6.1 \mathrm{E}-03$ & $1.3 \mathrm{E}-03$ & $2.8 \mathrm{E}-08$ & 3.7E-08 & $2.52 \mathrm{E}-08$ & 2.3E-08 & 0.93 & 0.02 & 11 & 6 \\
\hline A2261 & 260.61273 & 32.13266 & 0.331 & 0.004 & 4.7E-03 & $5.7 \mathrm{E}-04$ & $1.2 \mathrm{E}-07$ & 4.2E-08 & $1.25 \mathrm{E}-08$ & 9.4E-09 & 0.93 & 0.01 & 63 & 3 \\
\hline $1931-26$ & 292.95663 & -26.57594 & 0.545 & 0.006 & $1.7 \mathrm{E}-03$ & $6.2 \mathrm{E}-04$ & $3.5 \mathrm{E}-08$ & $2.8 \mathrm{E}-08$ & $6.56 \mathrm{E}-08$ & 2.3E-08 & 0.80 & 0.01 & -4 & 2 \\
\hline 2129-07 & 322.3573 & -7.69189 & 0.211 & 0.010 & 2.7E-02 & $2.8 \mathrm{E}-03$ & 7.0E-07 & $4.0 \mathrm{E}-07$ & $4.85 \mathrm{E}-08$ & $5.6 \mathrm{E}-08$ & 0.90 & 0.02 & 84 & 6 \\
\hline $2129+0005$ & 322.41548 & 0.08858 & 0.426 & 0.004 & $3.8 \mathrm{E}-03$ & $5.7 \mathrm{E}-04$ & $6.1 \mathrm{E}-08$ & $2.8 \mathrm{E}-08$ & $2.45 \mathrm{E}-08$ & $1.2 \mathrm{E}-08$ & 0.87 & 0.01 & 70 & 2 \\
\hline MS2137 & 325.0635 & -23.66098 & 0.589 & 0.007 & $2.5 \mathrm{E}-03$ & $7.9 \mathrm{E}-04$ & $5.1 \mathrm{E}-08$ & $5.7 \mathrm{E}-08$ & $5.60 \mathrm{E}-08$ & 3.7E-08 & 0.92 & 0.03 & 66 & 8 \\
\hline $2248-44$ & 342.18683 & -44.52922 & 0.194 & 0.003 & $1.5 \mathrm{E}-02$ & $9.7 \mathrm{E}-04$ & $2.0 \mathrm{E}-07$ & 7.1E-08 & $2.74 \mathrm{E}-08$ & $1.6 \mathrm{E}-08$ & 0.91 & 0.01 & 62 & 2 \\
\hline
\end{tabular}

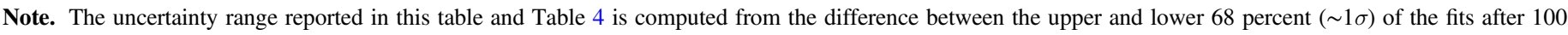
Monte Carlo runs, and the reported morphology value is the median value from those runs. AR is axis ratio; PA is Position Angle, in degrees east of north.

Table 4

X-Ray Morphological Properties for the CLASH Sample $0.5 R_{500}$

\begin{tabular}{|c|c|c|c|c|c|c|c|c|c|c|c|c|c|c|}
\hline Name & $\begin{array}{c}\text { R.A. } \\
\text { Centroid }\end{array}$ & $\begin{array}{c}\text { Decl. } \\
\text { Centroid }\end{array}$ & $C$ & $\begin{array}{c}C \\
\text { unc }\end{array}$ & $w$ & $\begin{array}{c}w \\
\text { unc }\end{array}$ & P30 & $\begin{array}{l}\mathrm{P} 30 \\
\text { unc }\end{array}$ & P40 & $\begin{array}{l}\text { P40 } \\
\text { unc }\end{array}$ & $\mathrm{AR}$ & $\begin{array}{l}\text { AR } \\
\text { unc }\end{array}$ & PA & $\begin{array}{l}\text { PA } \\
\text { unc }\end{array}$ \\
\hline A209 & 22.96919 & -13.6111 & 0.215 & 0.005 & $5.0 \mathrm{E}-03$ & $1.0 \mathrm{E}-03$ & $2.8 \mathrm{E}-08$ & 3.0E-08 & $1.9 \mathrm{E}-08$ & $1.8 \mathrm{E}-08$ & 0.88 & 0.02 & -46.2 & 2.5 \\
\hline A383 & 42.01382 & -3.52943 & 0.594 & 0.004 & $1.6 \mathrm{E}-03$ & 4.1E-04 & 5.2E-09 & 5.5E-09 & $1.4 \mathrm{E}-08$ & 8.2E-09 & 0.98 & 0.01 & -11.5 & 19.4 \\
\hline 0329-02 & 52.42257 & -2.1957 & 0.511 & 0.006 & $1.2 \mathrm{E}-02$ & $1.1 \mathrm{E}-03$ & $1.5 \mathrm{E}-07$ & $6.5 \mathrm{E}-08$ & 2.4E-08 & 1.8E-08 & 0.91 & 0.02 & -28.3 & 5.0 \\
\hline 0416-24 & $\ldots$ & & & $\ldots$ & & & & & & & & & & \\
\hline 0429-02 & 67.40009 & -2.88479 & 0.563 & $\ldots$ & 4.7E-03 & $1.1 \mathrm{E}-03$ & 4.4E-08 & 4.6E-08 & 9.3E-09 & $1.3 \mathrm{E}-08$ & 0.89 & 0.02 & -11.9 & 4.7 \\
\hline $0647+70$ & $\ldots$ & $\ldots$ & $\ldots$ & $\ldots$ & $\ldots$ & $\ldots$ & $\ldots$ & $\ldots$ & $\ldots$ & $\ldots$ & $\ldots$ & $\ldots$ & $\ldots$ & $\ldots$ \\
\hline $0717+37$ & $\ldots$ & $\ldots$ & $\ldots$ & $\ldots$ & $\ldots$ & $\ldots$ & $\ldots$ & $\ldots$ & $\ldots$ & $\ldots$ & $\ldots$ & $\ldots$ & $\ldots$ & $\ldots$ \\
\hline $0744+39$ & 116.21804 & 39.45748 & 0.375 & 0.007 & 2.3E-02 & $1.6 \mathrm{E}-03$ & $1.5 \mathrm{E}-07$ & $1.1 \mathrm{E}-07$ & 2.0E-07 & 8.2E-08 & 0.98 & 0.01 & -18.9 & 30.7 \\
\hline A611 & 120.23706 & 36.05736 & 0.394 & 0.004 & 2.4E-03 & 7.3E-04 & 7.9E-09 & $9.4 \mathrm{E}-09$ & 4.6E-09 & 4.8E-09 & 0.94 & 0.01 & 40.7 & 5.1 \\
\hline $1115+01$ & 168.96671 & 1.49809 & 0.481 & 0.008 & $5.3 \mathrm{E}-03$ & $1.8 \mathrm{E}-03$ & $8.8 \mathrm{E}-08$ & 7.7E-08 & $1.0 \mathrm{E}-08$ & 1.2E- 08 & 0.87 & 0.02 & -27.4 & 5.1 \\
\hline $1149+22$ & $\ldots$ & $\ldots$ & $\ldots$ & $\ldots$ & $\ldots$ & $\ldots$ & $\ldots$ & $\ldots$ & $\ldots$ & $\ldots$ & $\ldots$ & $\ldots$ & $\ldots$ & $\ldots$ \\
\hline A1423 & $\ldots$ & $\ldots$ & $\ldots$ & $\ldots$ & $\ldots$ & $\ldots$ & $\ldots$ & $\ldots$ & $\ldots$ & $\ldots$ & $\ldots$ & $\ldots$ & $\ldots$ & $\ldots$ \\
\hline $1206-08$ & 181.55361 & -8.80286 & 0.262 & 0.006 & $3.2 \mathrm{E}-02$ & $1.4 \mathrm{E}-03$ & $1.5 \mathrm{E}-06$ & $3.2 \mathrm{E}-07$ & 2.0E-07 & 7.2E-08 & 0.85 & 0.01 & 9.0 & 2.2 \\
\hline $1226+33$ & 186.74112 & 33.54693 & 0.315 & 0.011 & $6.8 \mathrm{E}-03$ & $1.6 \mathrm{E}-03$ & 4.0E-07 & $3.0 \mathrm{E}-07$ & $6.9 \mathrm{E}-08$ & 5.9E-08 & 0.93 & 0.02 & -86.3 & 10.5 \\
\hline $1311-03$ & 197.75701 & -3.17731 & 0.477 & 0.012 & $3.9 \mathrm{E}-03$ & $1.1 \mathrm{E}-03$ & 7.9E-08 & $1.0 \mathrm{E}-07$ & $1.0 \mathrm{E}-07$ & 9.1E-08 & 0.86 & 0.03 & -14.4 & 5.8 \\
\hline $1347-1145$ & 206.87867 & -11.75312 & 0.585 & 0.003 & $1.2 \mathrm{E}-02$ & 3.6E-04 & $5.1 \mathrm{E}-08$ & $1.4 \mathrm{E}-08$ & 1.4E-08 & 4.8E-09 & 0.85 & 0.01 & 0.4 & 1.2 \\
\hline $1423+24$ & 215.94964 & 24.07835 & 0.562 & 0.009 & 4.7E-03 & 1.4E-03 & $6.9 \mathrm{E}-08$ & 7.1E-08 & 3.1E-08 & 3.3E-08 & 0.89 & 0.03 & 18.0 & 7.0 \\
\hline $1532+30$ & 233.22436 & 30.34977 & 0.587 & 0.007 & $2.0 \mathrm{E}-03$ & 7.9E-04 & $2.0 \mathrm{E}-08$ & 2.0-E-08 & 7.7E-09 & 8.4E-09 & 0.93 & 0.02 & 51.7 & 9.0 \\
\hline $1720+35$ & 260.06927 & 35.60642 & 0.458 & 0.007 & $5.5 \mathrm{E}-03$ & $1.2 \mathrm{E}-03$ & $5.4 \mathrm{E}-08$ & 4.9E-08 & $2.0 \mathrm{E}-08$ & 2.0E-08 & 0.94 & 0.02 & 22.4 & 5.2 \\
\hline A2261 & 260.6115 & 32.13223 & 0.431 & 0.004 & 5.2E- 03 & 5.0E-04 & 5.7E-07 & 8.5E-08 & $2.1 \mathrm{E}-07$ & 3.4E-08 & 0.86 & 0.01 & 73.9 & 1.4 \\
\hline $1931-26$ & 292.95663 & -26.57591 & 0.575 & 0.005 & $1.8 \mathrm{E}-03$ & 5.9E-04 & 4.0E-08 & 2.7E-08 & $2.4 \mathrm{E}-08$ & 1.6E-08 & 0.79 & 0.01 & -4.2 & 1.8 \\
\hline 2129-07 & $\ldots$ & $\ldots$ & $\ldots$ & $\ldots$ & $\cdots$ & $\ldots$ & $\ldots$ & $\ldots$ & $\ldots$ & $\ldots$ & $\ldots$ & $\ldots$ & $\ldots$ & $\ldots$ \\
\hline $2129+00$ & 322.41523 & 0.08861 & 0.461 & 0.004 & $4.8 \mathrm{E}-03$ & 8.1E-04 & $3.9 \mathrm{E}-08$ & 2.2E-08 & 3.2E-08 & 1.4E-08 & 0.87 & 0.01 & 68.8 & 1.9 \\
\hline MS2137 & 325.06373 & -23.66072 & 0.612 & 0.007 & $5.4 \mathrm{E}-03$ & 9.9E-04 & $3.1 \mathrm{E}-08$ & $3.4 \mathrm{E}-08$ & $2.5 \mathrm{E}-08$ & 2.2E-08 & 0.97 & 0.03 & 60.3 & 7.3 \\
\hline $2248-44$ & 342.18634 & -44.52951 & 0.333 & 0.003 & $2.2 \mathrm{E}-02$ & 7.1E-04 & $1.8 \mathrm{E}-08$ & $1.3 \mathrm{E}-08$ & 5.2E-09 & 4.7E-09 & 0.91 & 0.01 & 55.2 & 2.3 \\
\hline
\end{tabular}

Note. Same convention as previous table. 

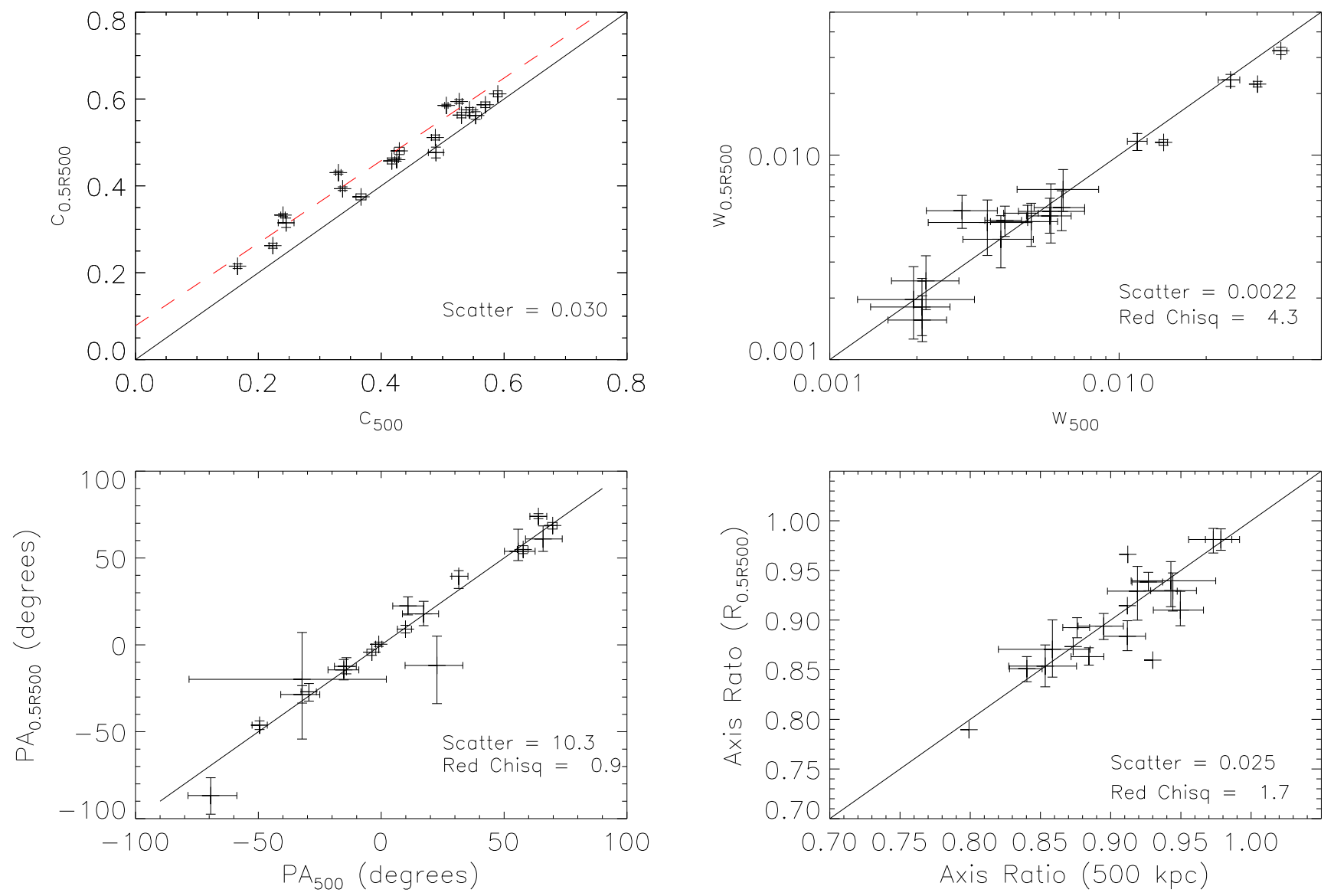

Figure 2. Comparison of X-ray measurements at $500 h_{70}^{-1} \mathrm{kpc}$ and at $0.5 R_{500}$, for the $19 / 25(19 / 20 \mathrm{X}$-ray selected) CLASH clusters for which direct comparison is possible. Solid black lines representing the equality line is overplotted for each. The scatter and reduced $\chi^{2}$ (Red Chisq) is in reference to that line for $w$, PA, and axis ratio. The best fit to the concentration measured at $500 \mathrm{kpc}$ vs. that at $0.5 R_{500}$ is plotted in a dashed red line in the upper left panel, and discussed in the text.

from one technique to another, we decided that the optimal choice was $500 \mathrm{kpc}$. For the clusters where we have measurements at both scales, with $R_{500}$ defined by the lensing measurements of Merten et al. (2015), we show in Figure 2 that measurements made at slightly different radii, unsurprisingly, are strongly correlated with each other. Except for concentration, the scatter in each case is computed relative to an identity line, not a fit. For concentration and centroid shift, the measurement errors are smaller than the scatter, but the typical variation is small in both cases, about $3 \%$ and $0.2 \%$ respectively. The dispersion in $\mathrm{PA}$ is about $6^{\circ}$ while the scatter in difference of the axis ratios is about $2 \%$, considerably less than the measurement uncertainty.

There is significant intrinsic scatter in the measurements in concentration, and this real variance is due to the direct dependence of the concentration parameter on how the outer radius is defined. Therefore when we compare concentration measured from X-ray maps to that measured on other maps, using the exact same inner and outer radius for the concentration ratio is important. The concentration defined at $0.5 R_{500}$ is strongly correlated with that computed at $500 \mathrm{kpc}$, such that a best linear fit to the measurements, including errors in $x$ and $y$, yields $C$ (at $\left.0.5 R_{500}\right)=0.95( \pm 0.01) C($ at $500 \mathrm{kpc})$ $+0.078( \pm 0.006)$. We plot this relation along with the line representing $C$ (at $\left.0.5 R_{500}\right)=C$ (at $500 \mathrm{kpc}$ ) in Figure 2. Figure 2 shows that the other parameters, centroid $w$, PAs, and axis ratios, can be measured in apertures of slightly different sizes without changing the nature of the correlation. We note that whenever we compare measurements from one technique to another for an individual cluster, we make that comparison over the identical region on the sky for each measurement.

In Table 2, we compare the mean and dispersion for morphological properties measured within $500 \mathrm{kpc}$ and within $0.5 R_{500}$ for the $\mathrm{X}$-ray selected subsample, as well as measurements made within $500 \mathrm{kpc}$ for the full sample. We note that the means and dispersions for properties measured for the X-ray selected CLASH subsample are virtually identical to each other, whether measured in the $500 \mathrm{kpc}$ aperture or within $0.5 R_{500}$. To test for radial variations, we repeated our morphological analysis at outer radii of 100, 200, 300, and $700 \mathrm{kpc}$ for our X-ray maps, and at 200, 300, and $700 \mathrm{kpc}$ for the lensing maps. We found no significant radial trends in measurements of ARs or PAs. The ARs remain within about $2 \%$ for all radii except for the innermost $\mathrm{X}$-ray radius at $100 \mathrm{kpc}$, where the AR is about $5 \%$ rounder than at $500 \mathrm{kpc}$. The averaged measured PAs vary very little with radius (in the $\mathrm{X}$-ray maps, the average difference was $\lesssim 10^{\circ}-15^{\circ}$ at radii between 200 and $700 \mathrm{kpc}$, and for the lensing maps the difference was $\lesssim 4^{\circ}$ ).

Therefore for this study we are comfortable with using the $500 \mathrm{kpc}$ aperture throughout, which is the only aperture common to all the clusters in the full range of measurement techniques considered in this paper. Note that the medians and standard deviations for the same quantity are similar across all samples except for the "SL" (lensing-selected) sample, which is constituted of the most obviously non-relaxed systems in the 
Table 5

Lensing Morphological Properties for the CLASH Sample $500 \mathrm{kpc}$

\begin{tabular}{|c|c|c|c|c|c|c|c|c|c|c|c|c|c|c|}
\hline Name & $\begin{array}{c}\text { R.A. } \\
\text { Centroid }\end{array}$ & $\begin{array}{c}\text { Decl. } \\
\text { Centroid }\end{array}$ & $C$ & $\begin{array}{c}C \\
\text { unc }\end{array}$ & $w$ & $\begin{array}{c}w \\
\text { unc }\end{array}$ & P30 & $\begin{array}{l}\text { P30 } \\
\text { unc }\end{array}$ & P40 & $\begin{array}{l}\mathrm{P} 40 \\
\text { unc }\end{array}$ & AR & $\begin{array}{l}\text { AR } \\
\text { unc }\end{array}$ & PA & $\begin{array}{l}\text { PA } \\
\text { unc }\end{array}$ \\
\hline A209 & 22.9688 & -13.6123 & 0.18 & 0.04 & $8.40 \mathrm{E}-03$ & 9E-03 & $6 \mathrm{E}-08$ & 4E-08 & $6 \mathrm{E}-08$ & $5 \mathrm{E}-07$ & 0.83 & 0.16 & -49 & 14 \\
\hline A383 & 42.01387 & -3.52979 & 0.31 & 0.09 & $2.96 \mathrm{E}-03$ & $6 \mathrm{E}-03$ & $6 \mathrm{E}-08$ & 2E-08 & $6 \mathrm{E}-08$ & $1 \mathrm{E}-07$ & 0.91 & 0.08 & 13 & 25 \\
\hline 0329-02 & 52.42153 & -2.19543 & 0.14 & 0.01 & $2.80 \mathrm{E}-02$ & $1 \mathrm{E}-02$ & 7E-07 & $6 \mathrm{E}-08$ & 7E-07 & 3E-08 & 0.84 & 0.07 & -36 & 9 \\
\hline 0416-24 & 64.03542 & -24.07298 & 0.13 & 0.03 & $2.68 \mathrm{E}-03$ & $3 \mathrm{E}-03$ & $5 \mathrm{E}-07$ & $3 E-07$ & $5 \mathrm{E}-07$ & 3E-07 & 0.78 & 0.13 & 42 & 4 \\
\hline 0429-02 & 67.40018 & -2.8851 & 0.20 & 0.08 & $1.13 \mathrm{E}-03$ & $2 \mathrm{E}-03$ & 2E-09 & $1 \mathrm{E}-08$ & 2E-09 & $8 \mathrm{E}-08$ & 0.78 & 0.15 & -8 & 3 \\
\hline $0647+70$ & 101.95882 & 70.24866 & 0.14 & 0.03 & 1.99E-03 & 4E-03 & $3 \mathrm{E}-08$ & $2 \mathrm{E}-07$ & $3 \mathrm{E}-08$ & $5 \mathrm{E}-07$ & 0.73 & 0.18 & -78 & 2 \\
\hline $0717+37$ & 109.386 & 37.7517 & 0.04 & 0.01 & $1.56 \mathrm{E}-03$ & 4E-03 & $2 \mathrm{E}-08$ & $3 \mathrm{E}-07$ & $2 \mathrm{E}-08$ & 2E-07 & 0.94 & 0.10 & 89 & 39 \\
\hline $0744+39$ & 116.21744 & 39.45835 & 0.19 & 0.06 & $3.90 \mathrm{E}-02$ & $2 \mathrm{E}-02$ & $3 \mathrm{E}-06$ & $2 \mathrm{E}-06$ & $3 \mathrm{E}-06$ & 7E-07 & 0.73 & 0.19 & -80 & 0 \\
\hline A611 & 120.23688 & 36.05659 & 0.19 & 0.07 & $1.62 \mathrm{E}-03$ & 9E-03 & 4E-09 & $8 \mathrm{E}-08$ & 4E-09 & $5 \mathrm{E}-08$ & 0.85 & 0.14 & 43 & 8 \\
\hline $1115+01$ & 168.96545 & 1.49939 & 0.15 & 0.04 & $9.32 \mathrm{E}-03$ & $5 \mathrm{E}-03$ & 2E-08 & 2E-09 & 2E-08 & 3E-09 & 0.87 & 0.07 & -41 & 5 \\
\hline $1149+22$ & 177.399 & 22.3991 & 0.08 & 0.00 & $9.52 \mathrm{E}-03$ & 2E-04 & $1 \mathrm{E}-07$ & $5 \mathrm{E}-08$ & $1 \mathrm{E}-07$ & 2E-08 & 0.95 & 0.00 & -34 & 0 \\
\hline A1423 & 179.32243 & 33.61049 & 0.31 & 0.09 & $1.43 \mathrm{E}-03$ & $5 \mathrm{E}-03$ & $1 \mathrm{E}-08$ & 4E-09 & $1 \mathrm{E}-08$ & 7E-08 & 0.71 & 0.28 & -88 & 30 \\
\hline 1206-08 & 181.55045 & -8.80092 & 0.13 & 0.00 & $3.01 \mathrm{E}-03$ & $8 \mathrm{E}-03$ & 3E-08 & $2 \mathrm{E}-07$ & 3E-08 & $1 \mathrm{E}-07$ & 0.80 & 0.09 & -77 & 2 \\
\hline $1226+33$ & 186.74152 & 33.54608 & 0.13 & 0.01 & $3.01 \mathrm{E}-02$ & $1 \mathrm{E}-02$ & $3 E-07$ & $1 \mathrm{E}-07$ & $3 E-07$ & $6 \mathrm{E}-08$ & 0.77 & 0.09 & 82 & 14 \\
\hline $1311-03$ & 197.758 & -3.17763 & 0.15 & 0.04 & $1.36 \mathrm{E}-03$ & $4 \mathrm{E}-03$ & 1E-09 & 2E-09 & $1 \mathrm{E}-09$ & $1 \mathrm{E}-08$ & 0.86 & 0.14 & 1 & 35 \\
\hline 1347-1145 & 206.879 & -11.753 & 0.14 & 0.04 & $1.41 \mathrm{E}-02$ & 9E-03 & 9E-08 & $6 \mathrm{E}-08$ & 9E-08 & $6 \mathrm{E}-08$ & 0.81 & 0.10 & 26 & 4 \\
\hline $1423+24$ & 215.94957 & 24.07864 & 0.17 & 0.06 & $1.91 \mathrm{E}-03$ & 9E-03 & 7E-09 & $8 \mathrm{E}-09$ & 7E-09 & $1 \mathrm{E}-07$ & 0.79 & 0.14 & 26 & 2 \\
\hline $1532+30$ & 233.22498 & 30.35002 & 0.19 & 0.06 & 8.91E-03 & $1 \mathrm{E}-02$ & $5 \mathrm{E}-08$ & 2E-08 & $5 \mathrm{E}-08$ & $1 \mathrm{E}-07$ & 0.84 & 0.11 & 39 & 18 \\
\hline $1720+35$ & 260.06976 & 35.60713 & 0.25 & 0.11 & $2.06 \mathrm{E}-03$ & 1E-02 & $8 \mathrm{E}-09$ & 2E-09 & $8 \mathrm{E}-09$ & 3E-07 & 0.74 & 0.21 & 11 & 3 \\
\hline A2261 & 260.61337 & 32.13261 & 0.22 & 0.03 & $1.06 \mathrm{E}-03$ & $3 \mathrm{E}-04$ & $1 \mathrm{E}-08$ & $1 \mathrm{E}-08$ & $1 \mathrm{E}-08$ & $1 \mathrm{E}-08$ & 0.89 & 0.08 & 42 & 3 \\
\hline $1931-26$ & 292.957 & -26.5758 & 0.18 & 0.03 & $5.56 \mathrm{E}-04$ & 7E-04 & 2E-09 & 4E-09 & 2E-09 & $5 \mathrm{E}-07$ & 0.70 & 0.18 & -3 & 4 \\
\hline 2129-07 & 322.35901 & -7.69128 & 0.15 & 0.04 & $2.05 \mathrm{E}-03$ & 4E-03 & 2E-08 & 9E-09 & $2 \mathrm{E}-08$ & $4 \mathrm{E}-07$ & 0.74 & 0.15 & 86 & 3 \\
\hline $2129+0005$ & 322.41678 & 0.08953 & 0.21 & 0.05 & $3.14 \mathrm{E}-03$ & 9E-03 & $5 \mathrm{E}-10$ & $7 \mathrm{E}-10$ & $5 \mathrm{E}-10$ & $6 \mathrm{E}-07$ & 0.68 & 0.23 & 68 & 2 \\
\hline MS2137 & 325.06329 & -23.65998 & 0.23 & 0.03 & $1.27 \mathrm{E}-02$ & $1 \mathrm{E}-03$ & 2E-08 & 2E-08 & 2E-08 & 7E-09 & 0.88 & 0.08 & 59 & 8 \\
\hline $2248-44$ & 342.18338 & -44.53075 & 0.15 & 0.04 & $9.49 \mathrm{E}-04$ & $1 \mathrm{E}-02$ & $5 \mathrm{E}-08$ & $4 \mathrm{E}-07$ & $5 \mathrm{E}-08$ & $8 \mathrm{E}-07$ & 0.71 & 0.17 & 53 & 9 \\
\hline
\end{tabular}

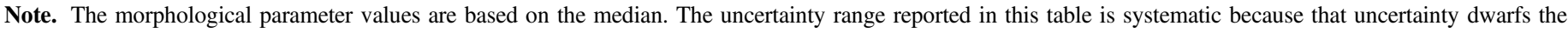

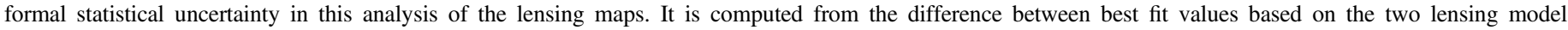
assumptions discussed in the text. The units and parameters in this table are the same as for the previous two tables.

CLASH sample. We summarize the comparison here: the X-ray images of the X-ray-selected CLASH sample are more centrally concentrated $(\sim 0.4$ versus $\sim 0.1)$, have smaller centroid shifts $(w \sim 0.005$ versus $w \sim 0.02)$, more circular (AR $\sim 0.9$ versus $\sim 0.8$ ), and have higher moment ratios $P 30$ and $P 40$ about an order of magnitude smaller than X-ray images of clusters in the lensing-selected sample.

The concentration $C$ has been used as an inexpensive surrogate for identifying candidate cool core clusters (e.g., Santos et al. 2008, 2010; Semler et al. 2012). Cool core clusters tend to be more concentrated than non-cool core clusters, and this trend applies to the CLASH sample as well. The radial bounds for the definition of $C$ in this work differ somewhat from the definitions used for the studies by Santos and Semler ( $R_{\text {inner }}-R_{\text {outer }}$ are $40-400 \mathrm{kpc} \mathrm{h}_{70}^{-1}$ instead of $100-500 \mathrm{kpc} \mathrm{h}_{70}^{-1}$, but the chosen energy range is very similar to that used by Santos et al. (2010) (we use a lower bound of $0.7 \mathrm{keV}$ rather than $0.5 \mathrm{keV}$ ). If we classify a cool-core cluster as having a central gas entropy $K_{0}$ of $k T_{e}^{-2 / 3} \sim 30 \mathrm{keV} \mathrm{cm}^{2}$ (e.g., Cavagnolo et al. 2009), the approximate threshold between cool-core clusters and non-cool core clusters in CLASH is $C \sim 0.4$, defined using the central gas entropies reported by Donahue et al. (2015). (The result is insensitive to whether we define cool cores by their central gas entropy or their cooling

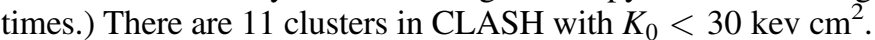
All eleven have $C<0.4$. Only one cluster has a higher central entropy and similar surface brightness concentration, MACSJ1311-03, with $K_{0}=47 \pm 6 \mathrm{keV} \mathrm{cm}^{2}$ and $C=$ $0.49 \pm 0.02$. We see no correlation between $C$ and $K_{0}$ for the low $K_{0}$ systems.

\subsection{Lensing Morphological Parameter Estimates}

Using the lensing maps, we computed the centroid shift, AR and PA. We did not compute power ratios for the lensing data since the uncertainties did not yield interesting results. We utilized otherwise identical procedures for quantifying the morphological parameters for the lensing maps.

As discussed in Section 3.3, the uncertainties for morphological parameters derived from lensing maps was estimated based on the differences between the morphology measurements made from the two different techniques. We verified that those systematic uncertainties are larger than the statistical estimates obtained from re-measuring 100 statistically resampled maps, and reflect a better quantification of the uncertainties of the estimates. Our results for deriving morphological parameters from the lensing-based surface mass density maps for individual clusters are reported in Table 5.

\subsection{SZE Morphological Parameter Estimates}

We computed the concentration, centroid shift, AR, and PA of the SZE images using the same procedures listed in Section 4.1. However, due to the limited spatial dynamic range of the SZE images, along with their modest $\mathrm{S} / \mathrm{N}$, we did not compute the value of the power ratios. In all cases, the same center positions and outer radii used for the X-ray and lensing analyses were also used for the SZE analysis.

The relatively large size of the Bolocam PSF produces a bias in some of the derived morphological parameters, particularly the value of the concentration and centroid shift. We correct for this bias using mock SZE observations of the simulated clusters 
Table 6

SZE Morphological Properties for the CLASH Sample $500 \mathrm{kpc}$

\begin{tabular}{|c|c|c|c|c|c|c|c|c|c|c|}
\hline Name & $\begin{array}{r}\text { R.A. } \\
\text { Centroid }\end{array}$ & $\begin{array}{r}\text { Decl. } \\
\text { Centroid }\end{array}$ & $C$ & $\begin{array}{r}C \\
\text { unc }\end{array}$ & $w$ & $\begin{array}{r}w \\
\text { unc }\end{array}$ & $\mathrm{AR}$ & $\begin{array}{l}\mathrm{AR} \\
\text { unc }\end{array}$ & PA & $\begin{array}{l}\text { PA } \\
\text { unc }\end{array}$ \\
\hline A209 & 22.9705 & -13.6121 & 0.087 & 0.009 & $2.9 \mathrm{E}-03$ & $3.9 \mathrm{E}-03$ & 0.93 & 0.03 & -20.8 & 12.6 \\
\hline A383 & 42.0142 & -3.5302 & 0.057 & 0.005 & $-2.0 \mathrm{E}-04$ & $3.4 \mathrm{E}-03$ & 0.98 & 0.01 & -8.0 & 43.4 \\
\hline $0416-24$ & 64.0369 & -24.0708 & 0.054 & 0.013 & $9.2 \mathrm{E}-03$ & 7.4E-03 & 0.93 & 0.05 & 13.1 & 15.6 \\
\hline 0429-02 & 67.3995 & -2.8854 & 0.049 & 0.009 & $3.4 \mathrm{E}-03$ & $4.5 \mathrm{E}-03$ & 0.95 & 0.04 & -43.5 & 15.4 \\
\hline $0647+70$ & 101.9593 & 70.2492 & 0.050 & 0.011 & $2.7 \mathrm{E}-03$ & $5.5 \mathrm{E}-03$ & 0.93 & 0.04 & $\cdots$ & \\
\hline A611 & 120.2345 & 36.0534 & 0.112 & 0.016 & $1.6 \mathrm{E}-02$ & 8.3E-03 & 0.97 & 0.04 & 43.6 & 42.8 \\
\hline $1115+01$ & 168.9666 & 1.5000 & 0.065 & 0.010 & $5.0 \mathrm{E}-03$ & $5.8 \mathrm{E}-03$ & 0.92 & 0.04 & -22.9 & 21.5 \\
\hline $1149+22$ & 177.3988 & 22.3989 & 0.027 & 0.010 & $3.7 \mathrm{E}-03$ & $4.5 \mathrm{E}-03$ & 0.96 & 0.03 & -50.1 & 25.7 \\
\hline A1423 & 179.3149 & 33.6146 & 0.099 & 0.029 & $4.1 \mathrm{E}-02$ & $1.6 \mathrm{E}-02$ & 0.65 & 0.14 & 61.0 & 14.9 \\
\hline $1206-08$ & 181.5508 & -8.8010 & 0.088 & 0.010 & $4.2 \mathrm{E}-03$ & $3.9 \mathrm{E}-03$ & 0.88 & 0.04 & -58.2 & 14.6 \\
\hline $1226+33$ & 186.7416 & 33.5480 & $\ldots$ & $\ldots$ & 2.6E-02 & $8.1 \mathrm{E}-03$ & 0.94 & 0.04 & 19.3 & 34.1 \\
\hline $1720+35$ & 260.0695 & 35.6081 & 0.054 & 0.008 & $6.3 \mathrm{E}-03$ & $3.6 \mathrm{E}-03$ & 0.94 & 0.03 & 89.8 & 7.7 \\
\hline A2261 & 260.6082 & 32.1355 & 0.116 & 0.020 & $3.2 \mathrm{E}-02$ & $1.0 \mathrm{E}-02$ & 0.85 & 0.09 & 68.1 & 18.3 \\
\hline $1931-26$ & 292.9564 & -26.5751 & 0.051 & 0.010 & $-4.1 \mathrm{E}-03$ & $6.5 \mathrm{E}-03$ & 0.88 & 0.04 & 0.9 & 9.7 \\
\hline 2129-07 & 322.3583 & -7.6925 & 0.081 & 0.014 & $1.1 \mathrm{E}-02$ & $5.5 \mathrm{E}-03$ & 0.94 & 0.03 & 29.7 & 44.2 \\
\hline $2129+00$ & 322.4146 & 0.0907 & 0.097 & 0.018 & $-8.0 \mathrm{E}-04$ & $1.3 \mathrm{E}-02$ & 0.90 & 0.06 & 28.5 & 18.5 \\
\hline MS2137 & 325.0622 & -23.6617 & 0.105 & 0.028 & $-1.8 \mathrm{E}-02$ & $1.5 \mathrm{E}-02$ & 0.84 & 0.12 & -80.6 & 19.6 \\
\hline $2248-44$ & 342.1839 & -44.5308 & 0.091 & 0.015 & $1.4 \mathrm{E}-03$ & $5.4 \mathrm{E}-03$ & 0.94 & 0.04 & -62.6 & 13.7 \\
\hline
\end{tabular}

Note. SZE morphological parameters computed as described in Section 4.3. No concentration is listed for $1226+33$ (the most distant in CLASH at $z \sim 0.9$ ) because $100 \mathrm{kpc}$ is small compared to the Bolocam PSF for this cluster. Three clusters do not have well-constrained PA values, and PA therefore are not reported for those clusters.

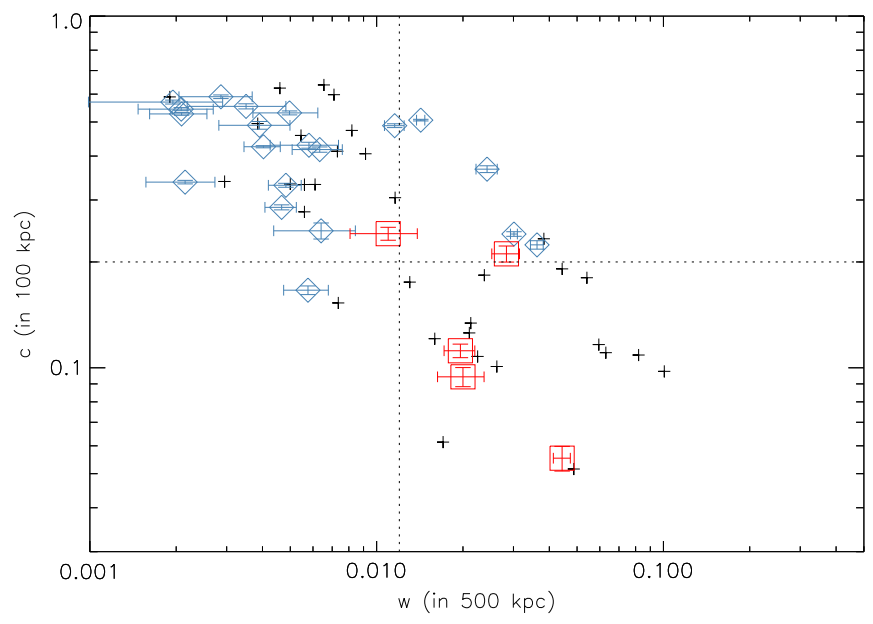

Figure 3. X-ray concentration is inversely correlated with centroid shift. Crosses are points from Cassano et al. 2010 (C10). Blue diamonds/red squares are for CLASH X-ray selected/lensing-selected clusters. Dashed lines are lifted approximately from $\mathrm{C} 10$. The lower right hand quadrant marks out the more disturbed clusters, while the upper left hand quadrant is occupied by more relaxed clusters.

from the MUSIC-2 sample according to the following procedure. First, we compute the true values of the morphological parameters using the mock SZE observations at the native resolution of the simulation. Next, the mock SZE observations are convolved with the Bolocam PSF, and the morphological parameters are recomputed. We then perform a linear fit to the true parameter values derived from the unconvolved mock observations and the (in general) biased parameter values derived from the PSF-convolved mock observations. A separate linear fit is performed for the clusters within each of the four redshifts of the MUSIC-2 sample $(0.250,0.333,0.429$, and 0.667). These linear fits, interpolated to the redshift of each real cluster in the CLASH sample, are then used to correct for the PSF-induced bias in the morphological parameters derived from the Bolocam data. In addition, the scatter in the mock-observation-derived values relative to the linear fits is added as a systematic uncertainty to all of the SZE results.

The SZE images contain noise that is correlated among pixels, and noise on large angular scales produces features that mimic deviations from spherical symmetry. In order to correct for this noise-induced bias we compute the value of the morphological parameters for each of the 1000 bootstrap noise images. In the case of the centroid shift and the AR, the mean value determined from these noise fits is significantly different from the nominal values of 0 and 1 . Therefore, for these two parameters we correct the value derived from the actual Bolocam images according to the mean value derived from the bootstrap fits. In some cases, due to noise fluctuations, this correction results in a best-fit parameter value outside of the physically allowed region. For example, a centroid shift that is less than, but statistically consistent with, zero.

The SZE-derived morphological properties within $500 \mathrm{kpc}$ apertures are listed in Table 6. 

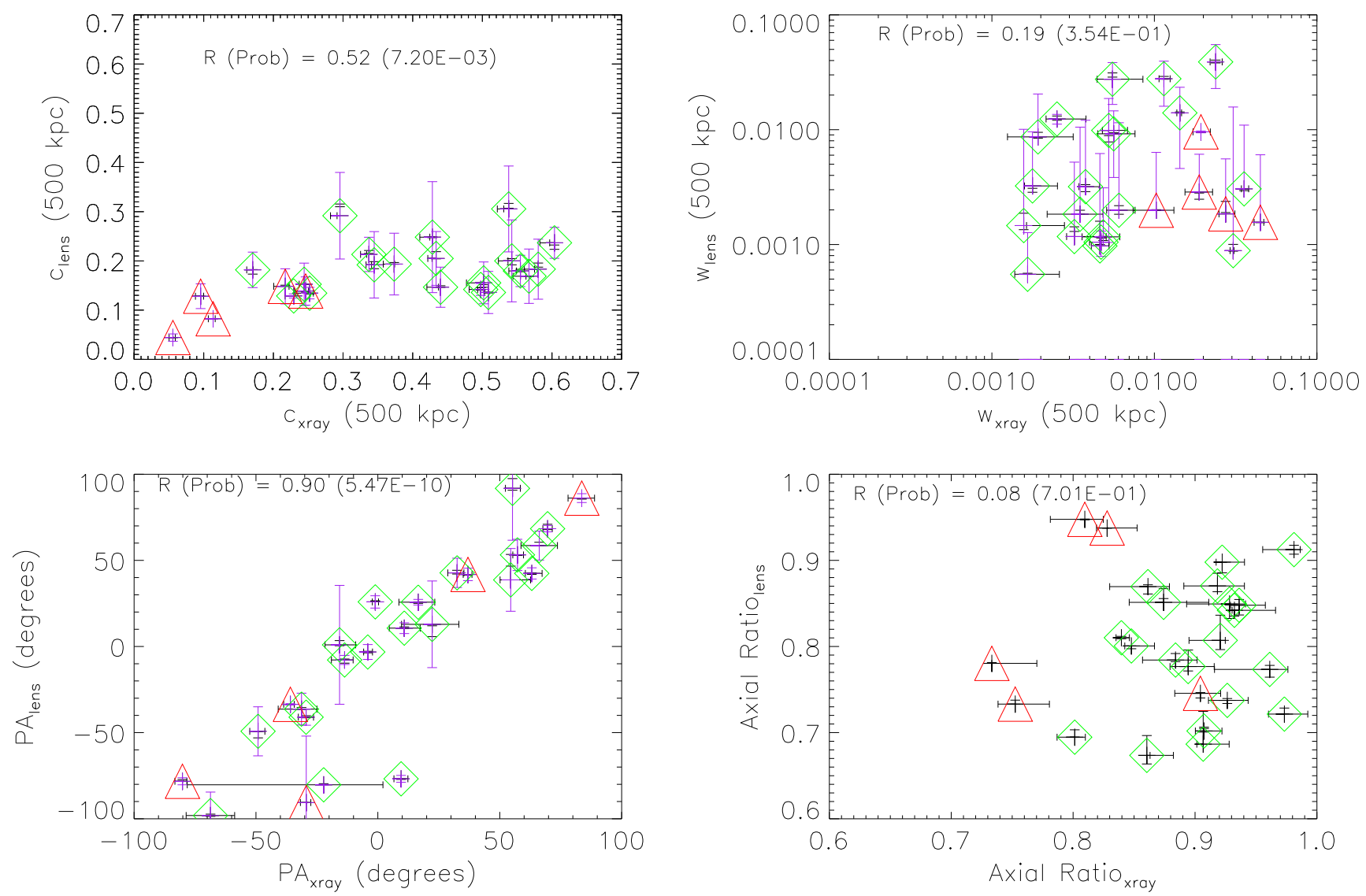

Figure 4. Morphological properties measured with $r_{\text {outer }}=500 h_{70}^{-1} \mathrm{kpc}$ for X-ray and lensing maps. The X-ray selected subsample is plotted with green diamonds. The lensing-selected subsample is plotted with red triangles. The black error bars are statistical. The systematic uncertainty for the lensing estimates are represented by the purple error bars. These uncertainties are based on the difference in results between the analyses of lensing maps from two different methods in Zitrin et al. (2015). Spearman's rank coefficient and probability (lower probabilities are more significant) were computed and reported in each plot for the full sample.

\section{DISCUSSION}

\subsection{X-Ray Morphology Correlations}

In general, we would expect that clusters with smaller X-ray concentration $(C)$, larger centroid shift $(w)$, and larger power ratios would be more likely to be disturbed clusters, a trend that can be seen in Table 2 and summarized in Section 4.2. We also might expect these measures to be loosely correlated with each other. Inspection of the CLASH morphologies plotted in Figure 3 shows the expected qualitative correlations seen in the $w-C$ diagram by Cassano et al. (2010) in their sample. The quadrants in this figure were defined by Cassano et al. (2010). The X-ray selected CLASH clusters are mostly relaxed in Figure 3. Fourteen of the $20 \mathrm{X}$-ray selected clusters lie in the upper-left quadrant, and none are located in the lower-right quadrant, which is where the most-disturbed clusters are.

We see that the X-ray morphologies of CLASH clusters show similar correlation between morphological parameters as seen in Cassano et al. (2010). The CLASH clusters that were lensing-selected have morphological indications that they are disturbed systems, similar to clusters in the Cassano sample that showed evidence for merger activity from the presence of radio halos and X-ray indicators.

\subsection{Comparison Between $X$-Ray and Lensing and SZE Morphologies}

We have compared the morphological parameters from X-ray and lensing maps in Figure 4 and the SZE maps in
Figure 5. Only the morphological property of PA correlates across all of the map classes. The lensing-X-ray PA correlation has only one distinct outlier, MACS1206. The discrepancy for this cluster disappears if its X-ray PA is measured at slightly smaller or larger radii. For all other clusters, the X-ray (or lensing) PA is not sensitive to choice of measurement radius for radii larger than $200 \mathrm{kpc}$. The other apparent outliers have large uncertainties. MACS0744 has a very uncertain X-ray PA, and MACS0717, a highly irregular system, has large systematic uncertainties in the determination of the orientation in its lensing map (as do CL1226 and Abell 1423, to a somewhat lesser extent.) But for the majority of the CLASH clusters, the lensing PA at $500 \mathrm{kpc}$ is quite similar to the one inferred from the X-rays.

The situation is much noisier in the SZ-X-ray comparison, due mainly to the larger PSF and lower S/N in the SZE maps. There is a clear correlation between the SZE and X-ray PA values, although there are also several statistically significant outliers. The cause of these outliers may be physical in nature, for example due to a difference in the gas orientation between the central region where most of the $\mathrm{X}$-ray signal originates and the outer regions near $500 \mathrm{kpc}$ where a large fraction of the SZE signal originates. Further, the presence and locations of shocks and/or high pressure regions could also produce differences in the SZE and X-ray measured PA values. However, the outliers may also be a result of systematics related to the SZE analysis. Although we have developed a rigorous procedure to correct for the large PSF and the large 

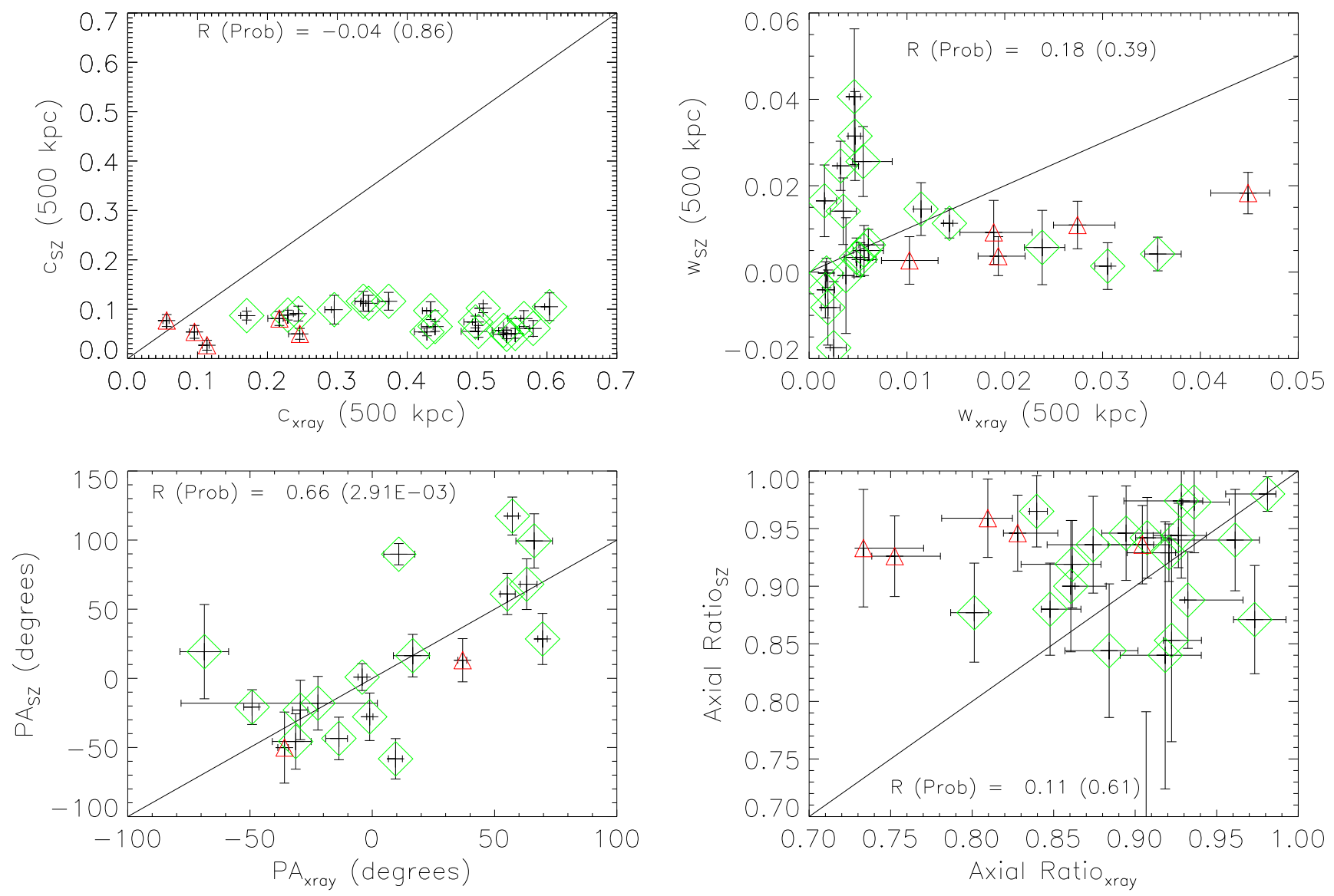

Figure 5. Morphological properties measured with $r_{\text {outer }}=500 h_{70}^{-1} \mathrm{kpc}$ for X-ray and SZE maps. The error bars for the SZE morphology are based on bootstrapped SZE maps as described in the text. Negative values for SZE $w$ arise from the correction prescription for the large angular scale noise, as described in the text. The $\mathrm{X}$-ray selected subsample is plotted with green diamonds. The lensing-selected subsample is plotted with red triangles. Spearman's rank coefficient and probability (lower probabilities are more significant) were computed and reported in each plot for the full sample. The PA is either unconstrained or poorly constrained in several of the SZE maps, and these clusters are not included in the plot.

angular scale noise in the SZE images, either or both of these effects could potentially bias our derived PA values.

Two effects could cause the dynamic range of the X-ray measurements of concentration and axis ratios to exceed those of the same properties measured from lensing and SZE maps.

1. The Chandra X-ray maps have an instrumental PSF with a width $\sim 1^{\prime \prime}$. The effective PSF is photon limited to be larger, however, the effective Chandra PSF for emissivity fluctuations is considerably more compact than the effective resolution of large scale structures in the lensing and SZE maps.

2. X-ray surface brightness scales like density squared, as opposed to linearly in gas density (or pressure) for the SZE signal and linearly in projected total mass density for the lensing signal. As a result, structures of higher density, such as the central region of the cluster, will produce an enhanced X-ray signal compared to the SZE or lensing signal.

We find the typical axis ratio for CLASH clusters in the $\mathrm{X}$-rays to be $0.88 \pm 0.06$, which is similar to the SZE maps at $0.90 \pm 0.06$, and somewhat more elongated (at $500 \mathrm{kpc}$ ) in lensing maps $0.80 \pm 0.08$ (although the LTM lensing models are more circular, at $0.92 \pm 0.04$.) In a one-to-one comparison, the lensing maps are more elongated than the projected X-ray emission, but are generally aligned in the same direction. That these clusters are typically circular is not surprising, since they were selected to be nearly circular in the X-ray. That they are similarly circular in SZ and lensing images then is also expected. That the gas is about $10 \%$ or so rounder than the projected mass at $500 \mathrm{kpc}$ was predicted by (e.g., Flores et al. 2007) when the total mass is dominated by collisionless dark matter, and thus the relative ARs (and therefore eccentricities) is consistent with gravitational potentials dominated by collisionless dark matter.

The SZE estimates of the axis ratios are not particularly correlated with the X-ray estimates at the same radius, but both estimates have a very similar mean and standard deviation, $0.9 \pm 0.06$, where the scatter is dominated by the measurement uncertainties. Abell 1423's SZE axis ratio is an outlier for the sample's range of SZE measurements, possibly due to the dim SZE signal toward this cluster. The two lowest X-ray axis ratios (i.e., the highest elongations) were found for MACS0416 and MACS0647. These are two of the five lensing-selected clusters from the CLASH survey (Postman et al. 2012a). Both of these CLASH clusters have evidence for interactions in their optical appearance (at least two bright galaxies in their core, with extended elongated intracluster light in between them).

We have plotted the histograms of centroid and PA differences in Figures 6 and 7. 

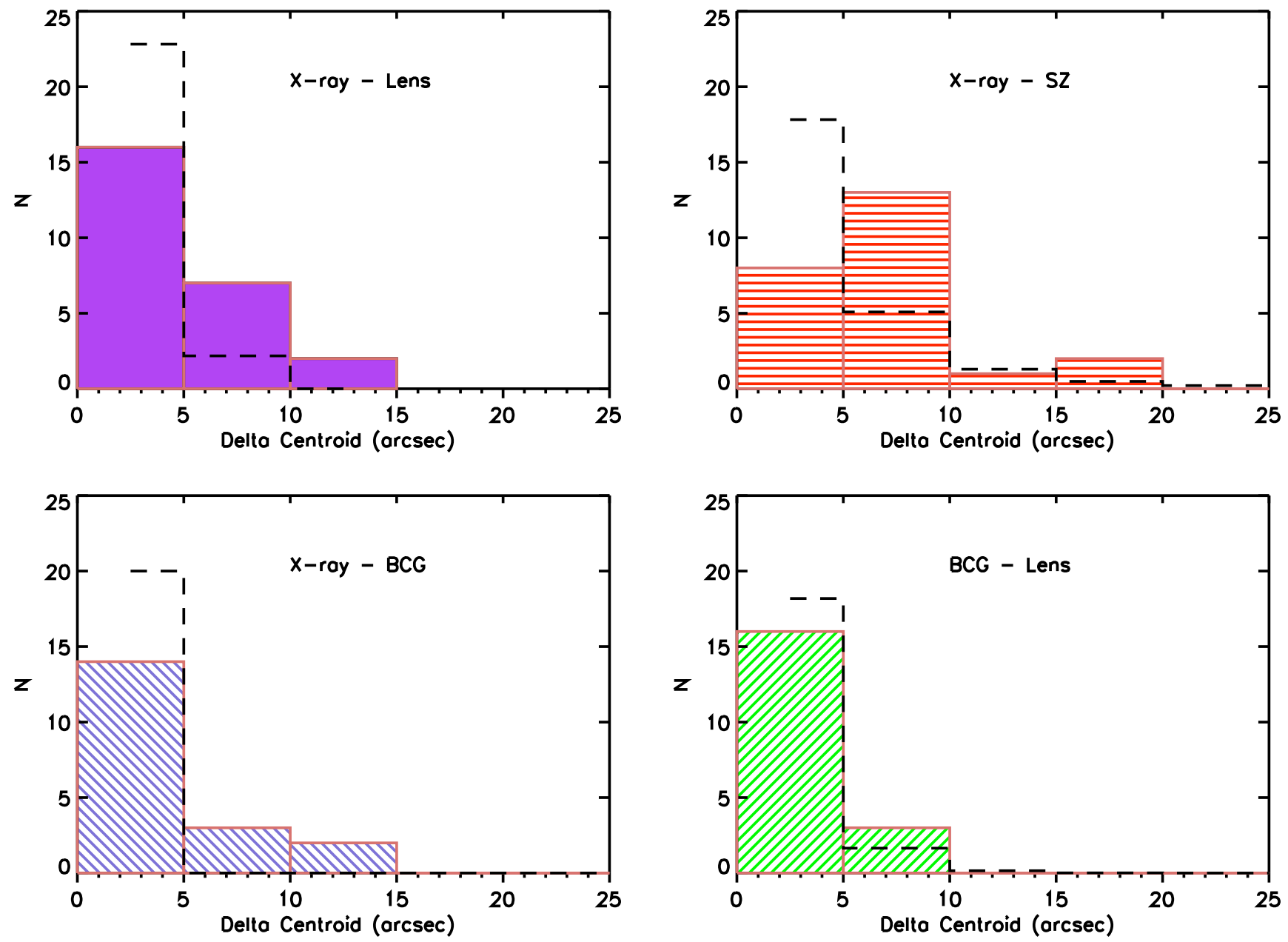

Figure 6. Histogram of centroid offsets (arcseconds). The dashed line shows the expected distribution of offsets based on measurement uncertainty if the true offset were identically zero.

\subsection{Comparison of X-Ray Morphological Properties of CLASH Clusters with Simulations}

In this section we compare the X-ray morphologies of CLASH clusters to the predicted X-ray morphology of clusters from the MUSIC simulation with similar masses and redshifts, but no morphological selection. For convenience, we choose to show the morphological parameters measured for the simulated clusters at $z=0.333$. We verified that the results are similar for the other redshifts. Each simulated cluster is represented three times in this sample, for 3 different viewing angles. Remember that in this work we are considering the complete set of simulated clusters selected only on the basis of the gravitational mass, and therefore the simulated clusters plotted in Figure 8 can represent any state of relaxation and virialization. For this reason, the expectation for the CLASH clusters is that their morphological parameters will be in the range of simulated clusters but skewed. In particular, we expect the CLASH clusters should be rounder (i.e., ARs near unity), and to have smaller $w$ and power ratios than mean values of the simulated clusters.

Scatter plots for the parameters $C, w, \mathrm{AR}$, and power ratios $P 30$ and $P 40$ are presented in Figure 8, in which one can see that the distribution of simulated clusters is similar to that of the observed CLASH clusters in the power ratios $P 30$ and $P 40$. The two power ratios are correlated and the 5 less-relaxed, lensing-selected CLASH clusters have larger power ratios than the 20-object X-ray selected CLASH subsample. On average, the CLASH clusters are rounder than the simulated clusters, in the sense that their ARs are closer to unity. This resultis not surprising, since the CLASH X-ray clusters were chosen in part for their round X-ray isophotes. However, even the irregular, lensing-selected clusters are rounder than most of the simulated clusters, according to the AR measurements: All of the lensingselected CLASH clusters have an AR about 0.8 , while only $15 \%$ of the simulated sample has an AR above 0.8 .

The centroid shifts $w$ of the CLASH clusters are smaller than most simulated clusters of similar mass, but they are not out of range: only $25 \%$ of the MUSIC clusters have $w \leqslant 0.01$ while $60 \%$ of the CLASH sample have such small $w$.

The range of concentration $C$ of the CLASH clusters is similar to that of the simulated sample. Note that a significant subset of simulated clusters have very high concentrations compared to those observed for CLASH clusters, even for the relaxed cool core clusters in CLASH. None of the simulated clusters have concentrations as low as a few of the CLASH strong-lensing selected clusters.

To summarize, compared to simulated clusters in MUSIC with a similar mass, CLASH cluster morphologies are on average rounder and have smaller centroid shifts. Their surface brightness concentrations and power ratios are similar to that of the mass-selected sample of simulated clusters. 

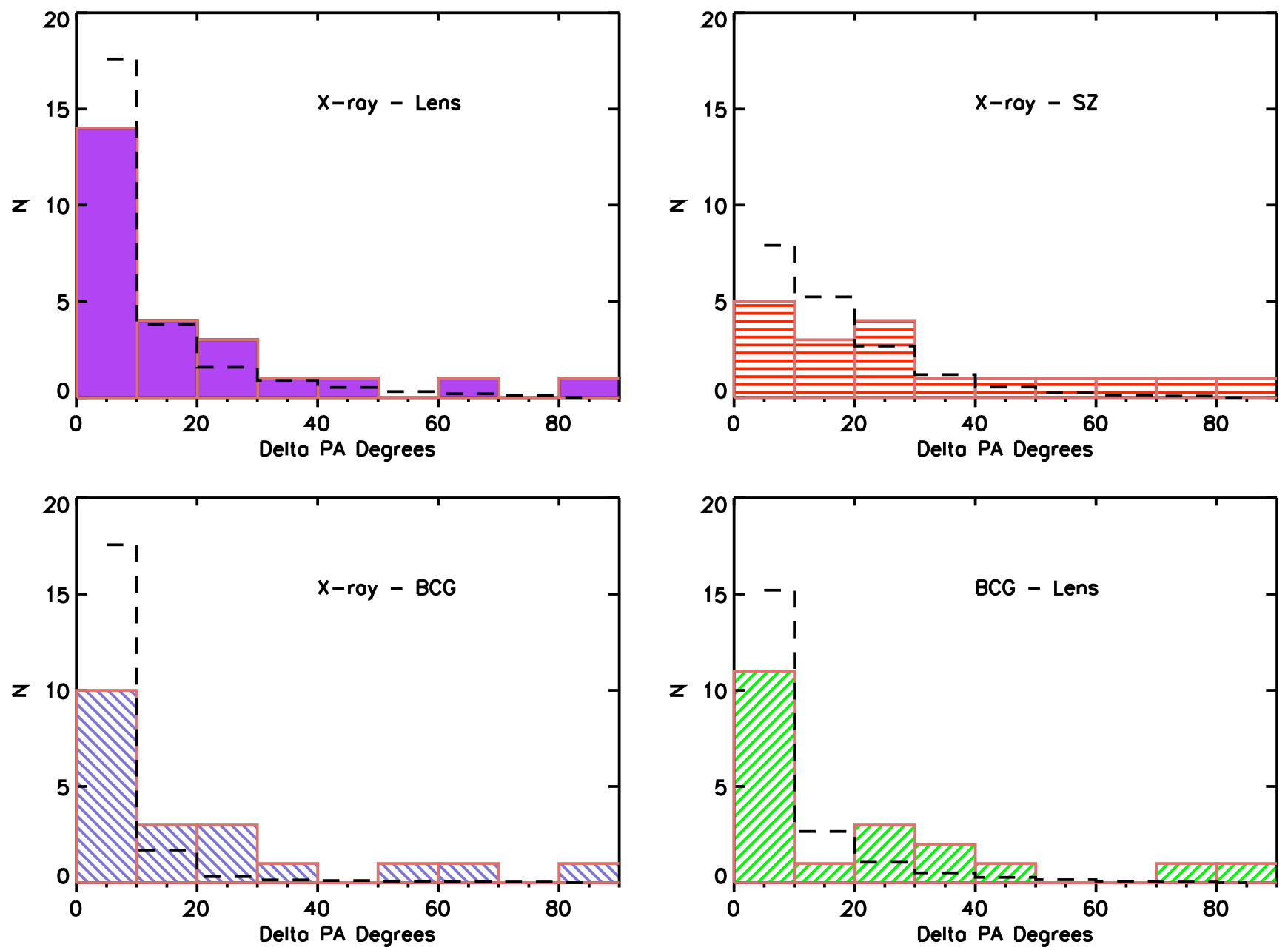

Figure 7. Histogram of PA offsets (degrees). The dashed line shows the expected distribution of offsets based on measurement uncertainty if the true offset were identically zero.

Meneghetti et al. (2014) defined regularity $M$ for a given simulated cluster in terms of the offsets of a set of morphological properties, in units of standard deviations. We refer the reader to Meneghetti et al. (2014) for details and specific relations, but we briefly review the results relevant to this work here. For each of five morphological parameters, $w$, eccentricity, $P 30$, and $P 40$, and $1 / C$, they find the difference between the log of the parameter and the log of the mean and divide by the standard deviation of the log quantities. They then sum these ratios to arrive at a composite regularity estimate $(M)$. The more negative $M$ is, the smaller $w, P 30$, and $P 40$, and the larger $C$ is compared to the full sample; the clusters with the most negative $M$ are generally rounder, more symmetric, and have higher central X-ray surface brightnesses compared to their outskirts. In addition, Meneghetti et al. (2014), define a simulated cluster as "relaxed" if the center of mass displacement from the minimum of the gravitational potential is small ( $\sim 0.07$ of the virial radius) and "superrelaxed" if in addition to a small displacement of the center of mass, the ratio between two times the kinetic and gravitational energy $(2 T /|U|)$ is nearly unity $(<1.35)$, and the mass in substructures is small $\left(<10 \% M_{\text {vir }}\right)$. They found no correlation between X-ray regularity $M$ and the fraction of non-relaxed or relaxed systems (see their Figure 15, lower panel). However, they saw a small correlation of $M$ with "super-relaxed" systems as one might expect: there are more "super-relaxed" clusters that are X-ray regular (negative $M$ ) than there are super-relaxed clusters that are X-ray irregular (positive $M$ ). For all simulated clusters, the mean ratio $2 T /|U|$ was $1.37 \pm 0.10$ and the mean center of mass offset was $0.08 \pm 0.05 R_{\mathrm{vir}}$ and the fractional mass in resolved substructures was $0.25 \pm 0.20$. For comparison, the same quantities for the simulated clusters chosen to match the CLASH clusters (as in Meneghetti et al. 2014) were $1.35 \pm 0.08,0.06 \pm 0.04 R_{\mathrm{vir}}$, and $0.21 \pm 0.13$, respectively. Thus, the relaxation measures of the complete, unabridged MUSIC runs and of the CLASH-like sub-sample are statistically similar, with differences in the means of a few percent, but always consistent at $1 \sigma$. The X-ray morphology of both the CLASH clusters and the simulated clusters are measured within $500 \mathrm{kpc}$ while any relaxation metric for a simulated cluster extends to the virial radius. A cluster can be $\mathrm{X}$-ray regular in its center while having substructure in its outskirts.

\section{BCG-CLUSTER MASS ALIGNMENTS}

BCGs are well-known for their alignment with the galaxy distribution of their host cluster of galaxies (e.g., Sastry 1968; Dressler 1978; Struble 1987; Tucker \& Peterson 1988) and with their X-ray emission (e.g., Allen et al. 1995; Hashimoto 

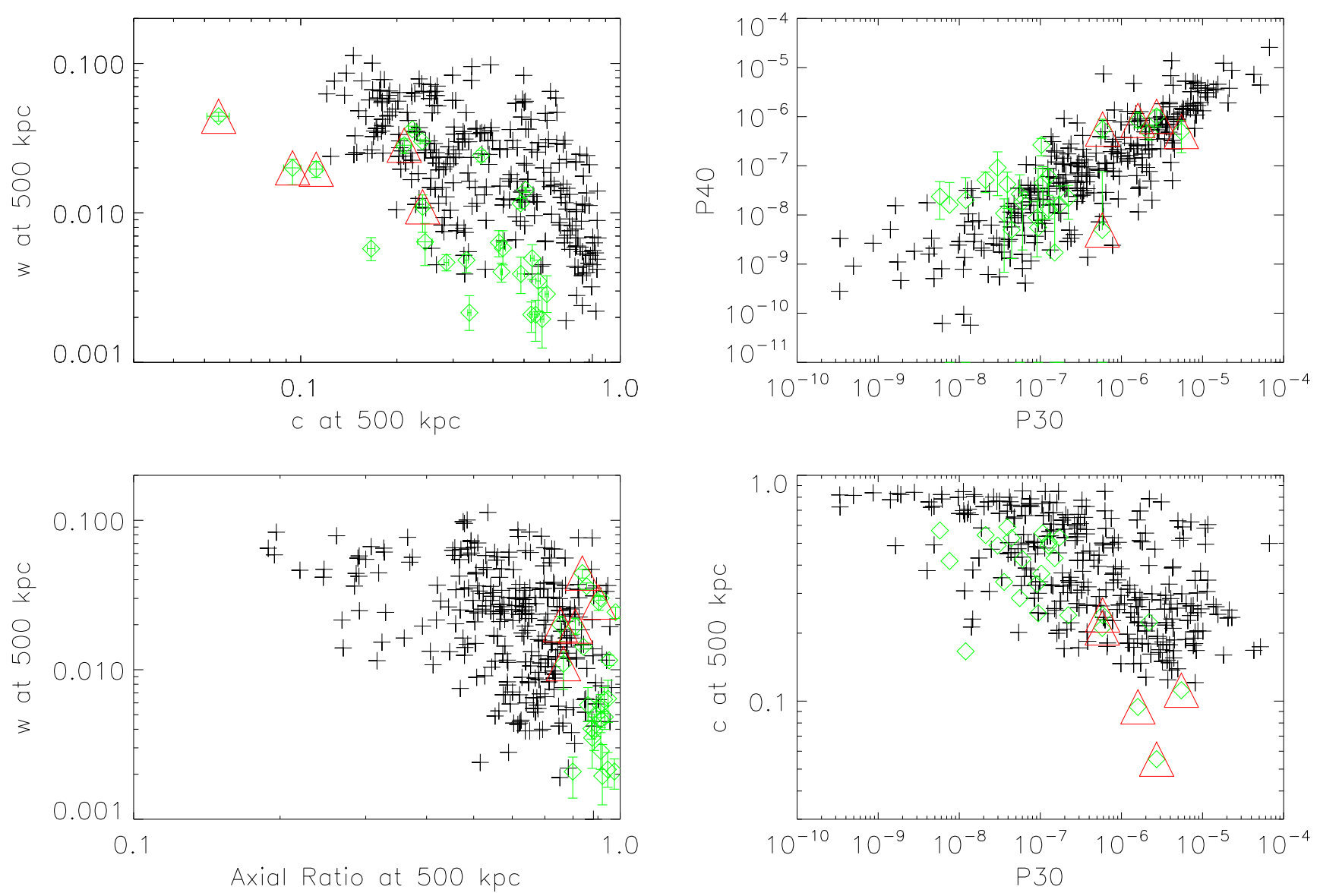

Figure 8. X-ray properties of simulated clusters from the MUSIC-2 simulation are plotted as black crosses. The CLASH X-ray morphological properties are plotted with green diamonds and error bars. The results for the five lensing-selected CLASH clusters are identified on each plot with red triangles. These clusters are expected to be less relaxed than the $20 \mathrm{X}$-ray selected clusters of the CLASH sample.

et al. 2008). This alignment indicates a connection between the galaxy-scale potential of the BCG and the larger cluster-scale potential. An early model of the formation of a BCG (West 1994) posits that the formation of the BCG is coupled to the formation of the cluster, and that the BCG stellar distribution retains a memory of the preferred accretion axis for the cluster itself. This explanation is viable and has survived observational tests such as Hashimoto et al. (2014) and Niederste-Ostholt et al. (2010). However, cosmological simulations of galaxycluster assembly still do not have enough spatial resolution to test this hypothesis, in that the spatial structure of the central galaxy on kpc scales is not well resolved in these large-volume simulations.

Our sample is not large enough or diverse enough to add much to what has already been discussed about the alignment of BCGs and their host clusters as seen in optical and X-ray light. However, the detailed lensing and SZE maps for this sample are new, and therefore we report here a distinct BCGcluster alignment effect between $10 \mathrm{kpc}$ scale PA measured from the stellar distribution from HST images of the BCG, and the gravitational potential measured at $500 \mathrm{kpc}$ by lensing, X-ray, and SZE maps for the BCGs in the CLASH sample. This correlation is significant even though these systems were chosen to be relatively round in their X-ray appearance.

Donahue et al. (2015) measured the PA and centroid of the near-infrared, rest-frame $1 \mu \mathrm{m}$ light in the CLASH BCGs in a similar fashion to the measurements presented in this work.
The radial scales of the measurements, derived from the analyses of $H S T$ WF3/NIR images were of order $10 \mathrm{kpc}$ for all 25 BCGs. The apertures were chosen to avoid contamination from other cluster galaxies and lensed features for quiescent BCGs and to overlap the regions of excess UV light in the others. The $1 \mu \mathrm{m}$ light from BCGs is dominated by light from stars, primarily old stars (5-10 billion years old). The gravitational mass in the centers of BCGs is also dominated by stars, so the starlight and the mass in the BCGs might be expected to be very well aligned.

The BCGs centroids align very well with the X-ray centroids of CLASH clusters, which is not surprising because good X-ray alignment with the BCG was a selection criterion for the 20 $\mathrm{X}$-ray selected clusters. The typical PA difference between the BCG and the X-ray PAs is $2^{\circ} \pm 24^{\circ}$, while between BCG and lensing PAs is $5^{\circ} \pm 25^{\circ}$. The PA difference between BCG and the cluster SZE PA is $38^{\circ} \pm 22^{\circ}$. The standard Spearman's test indicates a strong correlation in all 3 of these comparisons, where low probability indicates high significance of correlation: 0.89 (probability $=2 \times 10^{-7}$ ) for $\mathrm{X}$-ray $/ \mathrm{BCG}, 0.81$ (probability $=1.5 \times 10^{-7}$ ) for lensing $/ \mathrm{BCG}$, and 0.83 (probability $=2 \times 10^{-5}$ ) for SZE/BCG PAs. The 11 clusters with low central entropies $\left(K_{0}<30 \mathrm{keV} \mathrm{cm}^{2}\right)$, or cool core clusters, show less dispersion: $4 \pm 10,4 \pm 14$, and $33^{\circ} \pm 18^{\circ}$ respectively, for the X-ray, lensing and SZE- determined PAs. The correlation is somewhat less significant in the cool core sample because of the smaller number of clusters, but similarly 

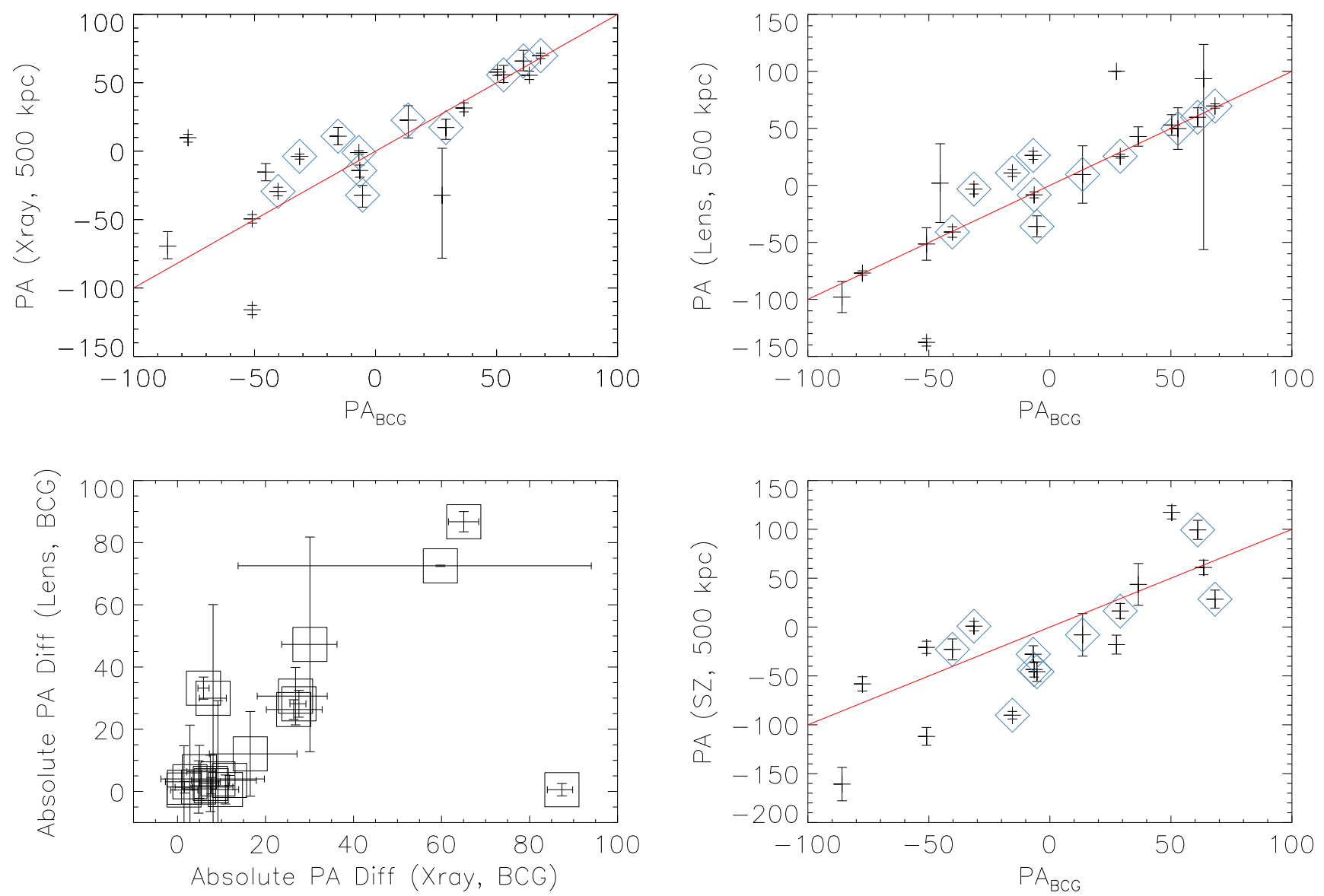

Figure 9. (Top row) Position angle in degrees of the near-IR emission of the BCG from Donahue et al. (2014) plotted against the PA for the X-ray and lensing maps at $500 \mathrm{kpc}$. (Lower left) The absolute value of the PA difference for the BCG, X-ray reference on the $x$-axis, while the lens PA reference is on the $y$-axis. The dispersion from zero offset is about $25^{\circ}$. The most significant outlier here is MACS1206, which has almost no offset between the PA measured in lensing and for the BCG, but the X-ray PA is almost perpendicular to those two. (Lower right) The SZE position angle in degrees plotted against the PA of the BCG.

strong $(r=0.79-0.75$ with probability $=0.004-0.007$ for $\mathrm{x}-$ ray-BCG and lens-BCG alignments respectively, while the SZ-BCG correlation drops to $r=0.60$ with probability $=0.07$, indicating a less than $2-\sigma$ correlation for PA in the CC-SZE BCG sample.

The offsets are correlated between lensing and X-ray comparisons, in that the BCGs with the largest X-ray PA offsets have the largest lensing PA offsets as well (Figure 9). The largest outliers in the X-ray/BCG comparison are Abell 2261 and MACS1206. Both of these clusters are BCGdominated, non-cool core systems. Abell 2261 is also a major outlier in the lensing/BCG PA comparison, while the BCG in MACS1206 is well-aligned with the lensing map. MACS0744 is the other significant outlier in the lensing/BCG PA comparison, (in X-rays, the PA for MACS0744 is not well determined.) MACS0744 is also a BCG-dominated, non-cool core cluster.

In summary, the PAs of the near-infrared light of BCGs aligns very well with the PAs of the X-ray gas maps, SZE maps, and the lensing projected mass maps. That alignment is not trivial, because of the factor of 50 difference in the radii where the PA is defined and compared for the BCG with that of the larger-scales of the cluster. The PA of the BCG is measured at a scale of $10 \mathrm{kpc}$ or less, and the PA of the gas and the projected mass were measured at a scale of $500 \mathrm{kpc}$. The correlation suggests that the mass distribution at $500 \mathrm{kpc}$ is strongly coupled to the mass distribution at $10 \mathrm{kpc}$, even in these relatively round and relaxed systems.

\section{CONCLUSIONS}

The CLASH project (Postman et al. 2012b) has collected a significant amount of data for a sample of 25 massive clusters of galaxies with redshifts from 0.2 to 0.9 , including strong and weak lensing constraints from HST (Merten et al. 2015), weak lensing constraints from Subaru (Umetsu et al. 2014), X-ray observations from Chandra and XMM (Donahue et al. 2014), and SZE observations from Bolocam (Czakon et al. 2015). To compare the results of the CLASH cluster sample to predictions from simulations, Meneghetti et al. (2014) selected simulated clusters replicating the morphological and temperature selection of the original CLASH sample. We present here the full uniformly measured X-ray morphological parameters and uncertainties for the 25 CLASH clusters used in that work. In addition we measure the same parameters in lensing and SZE maps. All parameters are measured inside radii of $500 \mathrm{kpc}$, and for a sub-sample of the clusters inside $0.5 R_{500}$. The full set of morphological properties are centroids, concentration ratios $(C)$, centroid shifts $(w)$, ARs, power ratios $\left(P_{3} / P_{0}, P_{4} / P_{0}\right)$, and PAs. We present means and standard deviations of these properties for the CLASH sample and for the X-ray selected CLASH subsample. For the first time we demonstrate a strong correlation between these morphological quantities as 
measured from lensing, X-ray, and SZE maps at a consistent radial scale of $500 h_{70}^{-1} \mathrm{kpc}$ (which is about half of $R_{500}$ for these clusters of galaxies).

In order to visualize how typical CLASH clusters are relative to a complete set of simulated clusters of similar mass, we compared high-mass clusters from the MUSIC simulations (Meneghetti et al. 2014) with our CLASH observations. The simulated clusters were mass-selected but are not matched in morphology as they were in Meneghetti et al. (2014). The full set of simulated clusters were expected to be more heterogeneous in structure and dynamic state in terms of relaxation than the CLASH sample. The CLASH clusters have similar power ratios, but $C, w$, and AR indicate that the CLASH clusters indeed appear more regular than a typical simulated cluster of similar mass. Very early attempts to simulate X-ray cluster morphologies typically failed to create clusters that were as relaxed as those selected from X-ray observations. The first simulations had limited cosmological context (simulations of individual clusters) or incorrect cosmology, such as a standard CDM universe with $\Omega_{M}=1$ which predicts more recent assembly activity, as discussed in (Tsai \& Buote 1996; Buote \& Xu 1997). Some early simulations assumed lower matter densities (Evrard et al. 1993; Mohr et al. 1995) and late time acceleration of the expansion (Thomas et al. 1998), but accurate predictions of the distribution of the hot gas inside clusters requires baryon prescriptions including the effects of AGNs and star formation feedback. These processes regulate the cooling and the heating of the cluster core and have significant impact on the central region. The morphological parameter most affected by baryon processes is the X-ray surface brightness concentration. However, AGN feedback may also change the shape and distribution of substructures because gas stripping becomes more efficient and merging systems are thermalized more quickly, potentially leading to changes in axis ratios. Future workers on cosmological simulations of clusters, expanding beyond what the CLASH team reported in Meneghetti et al. (2014), will be able to compare their simulations of high-mass clusters of galaxies to CLASH clusters by selecting a matched set of simulated clusters based on the measured morphologies we have reported here, and the global, core-excised gas temperatures reported in Postman (2012a).

We also show that the stellar mass of the BCG at small scales $(10 \mathrm{kpc})$, is strongly aligned with the PA of the matter distribution on much larger scales $(500 \mathrm{kpc})$, probed by lensing, X-ray, and SZE observations. To our knowledge, this the first time BCG PA has been demonstrated to align with the PA of the mass distribution in a galaxy-cluster sample with detailed lensing maps. However, the result has its roots in mid20th century astronomy. Alignments of BCGs with the galaxy distribution of their host clusters have been noted by Abell and others since the 1950 s.

This correlation shows that there is a strong relationship between the assembly of the mass distribution of the stars in the very center of the matter halo, inside the BCG, and the distribution of dark matter and the hot X-ray at large scales. The underlying cause of this correlation is likely to be the shape of the underlying gravitational potential. Cluster potential shapes are not expected to be perfectly spherical because in models of large scale structure matter does not flow in evenly over $4 \pi$ steradians but preferentially along filaments. Systematic alignment of BCGs with their clusters suggests that even the very inner regions of a galaxy cluster reflect the anisotropy of mass accretion on much larger scales.

M.D. was partially supported by an STScI/NASA award HST-GO-12065.07-A and NASA ADAP award NNX13AI41G. A.B. was partially supported by the same NASA ADAP award NNX13AI41G and a Chandra archive grant SAO AR3-14013X. E.R. was supported by FP7PEOPLE-2013-IIF (Grant Agreement PIIF-GA-2013-627474) and NSF AST-1210973. S.E. acknowledges the financial contribution from contracts ASI-INAF I/009/10/0 and PRIN-INAF 2012. This work was supported in part by National Science Foundation Grant No. PHYS-1066293 and the hospitality of the Aspen Center for Physics. J.S. was supported by NSF/AST-0838261, NASA/NNX11AB07G, and the Norris Foundation CCAT Postdoctoral Fellowship. N.G.C. was partially supported by a NASA Graduate Student Research Fellowship. The Bolocam observations were partially supported by the Gordon and Betty Moore Foundation. This research made use of the Caltech Submillimeter Observatory, which was operated at the time by the California Institute of Technology under cooperative agreement with the National Science Foundation (NSF/AST-0838261). G.Y. acknowledges financial support from MINECO's grant AYA2012-31101. The MUSIC simulations have been performed in the Marenostrum Supercomputer at the Barcelona Supercomputer Center, thanks to time granted by the Red Española de Supercomputación.

Facilities: Chandra X-ray Observatory, Hubble Space Telescope/ACS, Hubble Space Telescope/WFC3, Caltech Submillimeter Observatory.

\section{REFERENCES}

Abell, G. O. 1958, ApJS, 3, 211

Abraham, R. G., Valdes, F., Yee, H. K. C., \& van den Bergh, S. 1994, ApJ, 432, 75

Allen, S. W., Fabian, A. C., Edge, A. C., Bohringer, H., \& White, D. A. 1995, MNRAS, 275, 741

Anders, E., \& Grevesse, N. 1989, GeCoA, 53, 197

Bahcall, N. A. 1977, ARA\&A, 15, 505

Bautz, L. P., \& Morgan, W. W. 1970, ApJL, 162, L149

Beers, T. C., Geller, M. J., \& Huchra, J. P. 1983, ApJ, 264, 356

Binggeli, B. 1982, A\&A, 107, 338

Birkinshaw, M., Gull, S. F., \& Northover, K. J. E. 1978, MNRAS, 185, 245 Böhringer, H., Pratt, G. W., Arnaud, M., et al. 2010, A\&A, 514, A32

Borgani, S., \& Kravtsov, A. 2011, ASL, 4, 204

Buote, D. A., \& Tsai, J. C. 1995, ApJ, 452, 522

Buote, D. A., \& Tsai, J. C. 1996, ApJ, 458, 27

Buote, D. A., \& Xu, G. 1997, MNRAS, 284, 439

Carlstrom, J. E., Holder, G. P., \& Reese, E. D. 2002, ARA\&A, 40, 643

Cassano, R., Ettori, S., Giacintucci, S., et al. 2010, ApJL, 721, L82

Cavagnolo, K. W., Donahue, M., Voit, G. M., \& Sun, M. 2009, ApJS, 182,12

Comerford, J. M., \& Natarajan, P. 2007, MNRAS, 379, 190

Cuciti, V., Cassano, R., Brunetti, G., et al. 2015, A\&A, 580, A97

Czakon, N. G., Sayers, J., Mantz, A., et al. 2015, ApJ, 806, 18

D’Aloisio, A., \& Natarajan, P. 2011, MNRAS, 411, 1628

de Theije, P. A. M., Katgert, P., \& van Kampen, E. 1995, MNRAS, 273, 30

Donahue, M., Connor, T., Fogarty, K., et al. 2015, ApJ, 805, 177

Donahue, M., Voit, G. M., Mahdavi, A., et al. 2014, ApJ, 794, 136

Dressler, A. 1978, ApJ, 226, 55

Evrard, A. E., Mohr, J. J., Fabricant, D. G., \& Geller, M. J. 1993, ApJL, 419, L9

Fabricant, D. G., Kent, S. M., \& Kurtz, M. J. 1989, ApJ, 336, 77

Flores, R. A., Allgood, B., Kravtsov, A. V., et al. 2007, MNRAS, 377, 883

Forman, W., \& Jones, C. 1982, ARA\&A, 20, 547

Gardini, A., Rasia, E., Mazzotta, P., et al. 2004, MNRAS, 351, 505

Groener, A. M., Goldberg, D. M., \& Sereno, M. 2016, MNRAS, 455, 892

Hashimoto, Y., Henry, J. P., \& Boehringer, H. 2008, MNRAS, 390, 1562 
Hashimoto, Y., Henry, J. P., \& Boehringer, H. 2014, MNRAS, 440, 588

Host, O. 2012, MNRAS, 420, L18

Jeltema, T. E., Canizares, C. R., Bautz, M. W., \& Buote, D. A. 2005, ApJ, 624,606

Jones, C., \& Forman, W. 1984, ApJ, 276, 38

Jones, C., \& Forman, W. 1999, ApJ, 511, 65

Kent, S. M., \& Sargent, W. L. W. 1983, AJ, 88, 697

Kravtsov, A. V., Klypin, A. A., \& Khokhlov, A. M. 1997, ApJS, 111, 73

Lau, E. T., Nagai, D., Kravtsov, A. V., Vikhlinin, A., \& Zentner, A. R. 2012, ApJ, 755, 116

Mantz, A. B., Allen, S. W., Morris, R. G., et al. 2015, MNRAS, 449, 199

Maughan, B. J., Jones, C., Forman, W., \& Van Speybroeck, L. 2008, ApJS, 174,117

Meneghetti, M., Fedeli, C., Pace, F., Gottlöber, S., \& Yepes, G. 2010, A\&A, 519, A90

Meneghetti, M., Rasia, E., Vega, J., et al. 2014, ApJ, 797, 34

Merten, J., Meneghetti, M., Postman, M., et al. 2015, ApJ, 806, 4

Mohr, J. J., Evrard, A. E., Fabricant, D. G., \& Geller, M. J. 1995, ApJ, 447, 8

Morgan, W. W. 1961, PNAS, 47, 905

Navarro, J. F., Frenk, C. S., \& White, S. D. M. 1997, ApJ, 490, 493

Niederste-Ostholt, M., Strauss, M. A., Dong, F., Koester, B. P., \& McKay, T. A. 2010, MNRAS, 405, 2023

O’Hara, T. B., Mohr, J. J., Bialek, J. J., \& Evrard, A. E. 2006, ApJ, 639, 64

Plagge, T., Benson, B. A., Ade, P. A. R., et al. 2010, ApJ, 716, 1118

Poole, G. B., Fardal, M. A., Babul, A., et al. 2006, MNRAS, 373, 881

Postman, M., Coe, D., Benítez, N., et al. 2012a, ApJS, 199, 25

Postman, M., Lauer, T. R., Donahue, M., et al. 2012b, ApJ, 756, 159

Rasia, E., Borgani, S., Ettori, S., Mazzotta, P., \& Meneghetti, M. 2013a, ApJ, 776, 39

Rasia, E., Mazzotta, P., Bourdin, H., et al. 2008, ApJ, 674, 728

Rasia, E., Meneghetti, M., \& Ettori, S. 2013b, AstRv, 8, 40

Rasia, E., Meneghetti, M., \& Ettori, S. 2013c, AstRv, 8, 40

Santos, J. S., Rosati, P., Tozzi, P., et al. 2008, A\&A, 483, 35
Santos, J. S., Tozzi, P., Rosati, P., \& Böhringer, H. 2010, A\&A, 521, A64

Sastry, G. N. 1968, PASP, 80, 252

Sayers, J., Czakon, N. G., Mantz, A., et al. 2013, ApJ, 768, 177

Sembolini, F., Jahan Elahi, P., Pearce, F. R., et al. 2015, MNRAS, submitted (arXiv:1511.03731)

Sembolini, F., Yepes, G., De Petris, M., et al. 2013, MNRAS, 429, 323

Semler, D. R., Šuhada, R., Aird, K. A., et al. 2012, ApJ, 761, 183

Splinter, R. J., Melott, A. L., Linn, A. M., Buck, C., \& Tinker, J. 1997, ApJ, 479,632

Springel, V. 2005, MNRAS, 364, 1105

Struble, M. F. 1987, ApJ, 317, 668

Sunyaev, R. A., \& Zeldovich, Y. B. 1972, CoASP, 4, 173

Thomas, P. A., Colberg, J. M., Couchman, H. M. P., et al. 1998, MNRAS, 296, 1061

Tsai, J. C., \& Buote, D. A. 1996, MNRAS, 282, 77

Tucker, G. S., \& Peterson, J. B. 1988, AJ, 95, 298

Tyson, J. A., Wenk, R. A., \& Valdes, F. 1990, ApJL, 349, L1

Umetsu, K., Medezinski, E., Nonino, M., et al. 2014, ApJ, 795, 163

Umetsu, K., Zitrin, A., Gruen, D., et al. 2015, ApJ, submitted (arXiv:1507.04385)

Ventimiglia, D. A., Voit, G. M., Donahue, M., \& Ameglio, S. 2008, ApJ, 685,118

Vikhlinin, A., Burenin, R. A., Ebeling, H., et al. 2009, ApJ, 692, 1033

Vikhlinin, A., Kravtsov, A., Forman, W., et al. 2006, ApJ, 640, 691

Voit, G. M. 2005, RvMP, 77, 207

Voit, G. M., \& Bryan, G. L. 2001, Natur, 414, 425

West, M. J. 1994, MNRAS, 268, 79

Weymann, R. 1965, PhFl, 8, 2112

Wik, D. R., Sarazin, C. L., Ricker, P. M., \& Randall, S. W. 2008, ApJ, 680, 17

Zitrin, A., Fabris, A., Merten, J., et al. 2015, ApJ, 801, 44

Zwicky, F., Herzog, E., Wild, P., Karpowicz, M., \& Kowal, C. T. 1961, Catalogue of Galaxies and of Clusters of Galaxies, Vol. I (Pasadena: California Institute of Technology) 